\title{
Synthesis and Study of Many New Chalcone Derivatives
}

\author{
Natiq G. Ahmad Murtatha H. Ali \\ Chemistry Department, College of Education, University of \\ Mosul \\ Mosul - Iraq
}

Received

09/09/2013
Accepted

$06 / 11 / 2013$

\section{تحضير ودراسة عدد من مشتقات الجالكون الجديدة}

\section{الخلاصة}





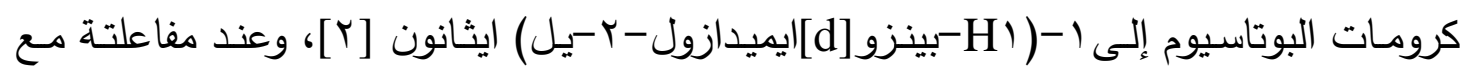

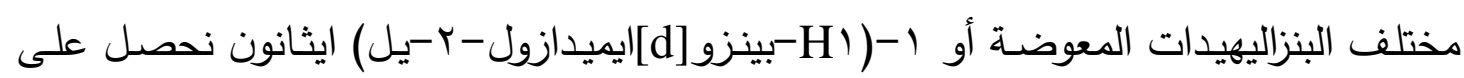

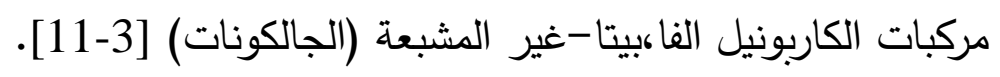









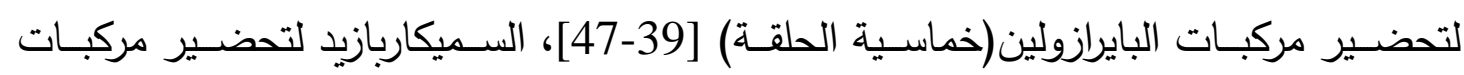

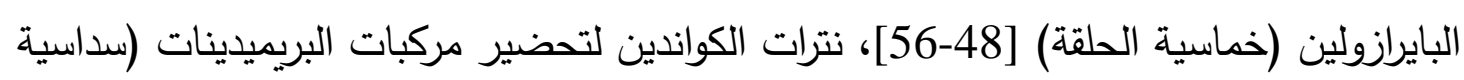
الحلقـة).657-67 اورثوفنيلين ثـائي الأمسين لتحضير مركبـات البنزودايازبين (سباعية الحلقـة)

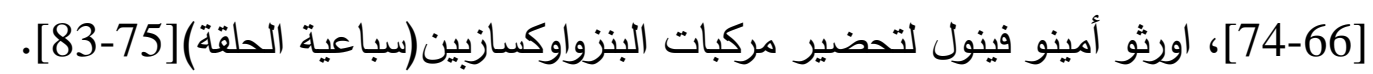

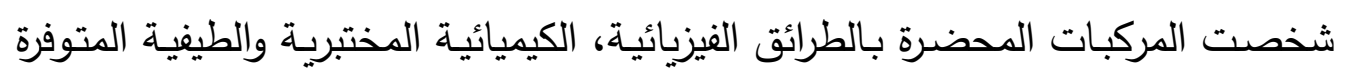
(درجة الانصهار ، التغيرات اللونية، طيف الاشعة تحت الحمراء، طيف الاشعة فوق البنفسجية، طيف الرنين النووي المغناطيسي وعدد من الحسابات النظرية).

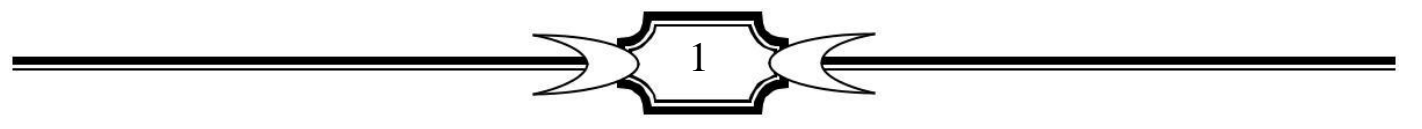




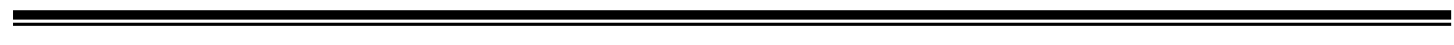

ABSTRACT

This study include the synthesis of the compound 1- $(1 \mathrm{H}-$ benzo[d]imidazol-2-yl)ethanol [1], from the reaction of ortho pheneylene diamine with lactic acid and then oxidized using potassium dichromate to give1-(1H-benzo[d]imidazol-2-yl) ethanone [2]. Reaction of this compound with different substituted benzaldehydes or 1-(1Hbenzo[d]imidazol-2-yl)ethanone, to obtain $\alpha, \beta$-unsaturated carbonyl compounds (chalcones) [3-11]. These chalcones have been used in preparing number of heterocyclic compounds through reaction with the hydrogen peroxide to get the oxiran compounds (three membered rings) [12-20], hydroxyl amine hydrochloride to prepare the isooxazoline compounds(five membered rings) [21-29], 3-nitrobenzohydrazide to prepare the pyrazoline compounds(five membered rings)[30-38], thiosemicarbazide hydrochloride to prepare the pyrazoline compounds(five membered rings) [39-47], semicarbazide to prepare the pyrazoline compounds(five membered rings) [48-56], guanidine nitrate to prepare the pyrimidine compounds(six membered rings) [57-65], ortho pheneylenediamine to prepare the benzodiazepine compounds (seven membered rings) [66-74], ortho aminophenol to prepare the benzoxazepine compounds(seven membered rings) [75-83].

The synthesized compounds were identified, using chemical, physical and spectral methods (melting points, color change, infrared spectra, ultraviolet spectra, nuclear magnetic resonance spectra and many theoretical studies).

\section{INTRODUCTION}

Chalcones constitute an important group of natural products and serve as precursors for the synthesis of different classes of flavonoids, which are common substances in plants. Chalcones are open-chain flavonoids in which two aromatic rings are joined by a three carbon $\alpha, \quad \beta,-$ ansaturated carbonyl system (1,3-diphenyl-2-propen-1-one $)^{[1]}$. Chalcone derivatives have received a great attention due to their relatively simple structure, and wide variety of pharmacological activities reported for these compounds include anti-inflammatory ${ }^{[2]}$, antiulcerative $^{[3]}$, analgesic ${ }^{[4]}$, anti-viral ${ }^{[5]}$, anti-malarial ${ }^{[6]}$, anti-fungal ${ }^{[7]}$ and anti-cancer activities ${ }^{[8]}$. Recently 1-(4- Floro phenyl)-3-(4-Methyl phenyl)-2-propen-1-one have been prepared at (2010) ${ }^{[9]}$ from the reaction between ( $p$-fluroacetophenone) and ( $\mathrm{p}$ - methylbenzaldehyde)in basic medium . The reaction of acetyl furan with 3,4,5-triethoxybenzaldehyde give the (chalcone) ${ }^{[10]}$ 1-(furan-2-yl)-3-(3,4,5-trimethoxyphenyl)-2propen-1-one. Also the chalcones was synthesized by using (Grinding Technique) $)^{[11]}$ 


\section{EXPERIMENTAL}

The melting points were measured on an electro thermal 9300 engineering LTD and are uncorrected, IR spectrum were recorded on infrared spectrophotometer model tensor 27, Bruker Co., Germany, using $\mathrm{KBr}$ discs. The U.V spectrum were recorded on UV-Visible Shimadzu 1601 spectrophotometer using DMSO as a solvent. The ${ }^{1}$ HNMR [400MHz, $\delta, \mathrm{ppm}, \mathrm{DMSO}-\mathrm{d}_{6}$ ] were recorded on Bruker : AvanceIII 400 $\mathrm{MHz}$ : Tocat / Gazi Osman pasa University(Turkey). All chemicals were purchased from Fluka and BDH chemical Ltd.

Synthesis of 1-(1H-benzo[d]imidazol-2-yl) ethanol [1] ${ }^{[12]}$

A mixture of $(0.05 \mathrm{~mol}, 5.4 \mathrm{~g})$ ortho phenylene diamine and $(0.05 \mathrm{~mol}, 4.5 \mathrm{~g})$ of lactic acid and $25 \mathrm{~mL}$ of $4 \mathrm{~N} \mathrm{HCl}$, was refluxed for 14 hours, then cooled to room temperature then the mixture was neutralize by adding $10 \% \mathrm{NaOH}$ solution. The precipitate was filtered, washed with cold water, dried and recrystallized from hot water. The yield of the reaction was $(93 \%)$.

Synthesis of 1-(1H-benzo[d]imidazol-2-yl) ethanone [2] $]^{[13,14]}$

To a solution of 1-(1H-benzo[d]imidazol-2-yl) ethanol $(0.01 \mathrm{~mol}$, $1.62 \mathrm{~g})$ in queous acetic acid $(25 \mathrm{~mL})$ was added at room temperature a solution of $\mathrm{K}_{2} \mathrm{Cr}_{2} \mathrm{O}_{7}(0.01 \mathrm{~mol}, 2.94 \mathrm{~g})$ in water $(20 \mathrm{~mL})$ and the mixture reflux for 3 hours. The reaction mixture was cooled and neutralized very carefully with aq. $\mathrm{NH}_{4} \mathrm{OH}$. The separated solid was filtered and washed with cold water, dried and purified by recrystallization from ethanol.

Synthesis of the chalcones ${ }^{[15]}$ (E)-1-(1H- benzo[d]imidazol-2-yl) -3(substituted phenyl)prop-2-en-1-one [3-9], (E)-1-(1H-benzo [d] imidazol-2yl)-3-(pyridine-3-yl)prop-2-en-1- one [3-10]

A mixture of 1-(1H-benzo[d]imidazol-2-yl) ethanon $(0.001 \mathrm{~mol}$, $0.16 \mathrm{gm})$ dissolved in ethanol $(25 \mathrm{~mL}), 10 \mathrm{~mL}$ of $10 \% \mathrm{KOH}$ and substituted aromatic aldehyde $(0.001 \mathrm{~mol})$ The reaction mixture was stirred at room temperature for $6 \mathrm{hrs}$. The reaction mixture was cooled and poured into ice water, neutralized with $30 \%$ acetic acid, the solid product was filtered then dried and recrystallized from ethanol.

Synthesis of the chalcone 1, 3-di (1H-benzo[d]imidazol-2-yl) prop2-en-1-one $[11]^{[16]}$

A mixture of 1-(1H-benzo[d]imidazol-2-yl) ethanone $(0.005 \mathrm{~mol}$, $0.8 \mathrm{~g})$ dissolved in ethanol $(20 \mathrm{~mL}), 10 \mathrm{~mL}$ of $10 \% \mathrm{KOH}$ The reaction mixture was reflux for $6 \mathrm{hrs}$. The reaction mixture was cooled and poured into ice water, neutralized using $30 \%$ acetic acid the solid products was filtered off then dried and recrystallized from ethanol.

Synthesis of [3-(1H-benzo[d]imidazol-2-yl) (3-(substituted phenyl) oxiran-2-yl) methanone [12-18], [ 1H-benzo[d]imidazol-2-yl) (3(pyridine-3-yl) oxiran-2-yl) methanone [19] and (3- (1H-benzo [d] 
imidazol-2-yl) -3- methyloxiran -2- yl) $(1 \mathrm{H}$-benzo [d] imidazol -2-yl) methanone $[20]^{[17]}$.

To a mixture of $(0.001 \mathrm{~mol})$ of one of the chalcones [3-11] dissolved in $(10 \mathrm{~mL})$ of ethanol, $(1 \mathrm{~mL})$ of $10 \%$ sodium hydroxide in $(10$ $\mathrm{mL}$ ) of $30 \%$ hydrogen peroxide were added drop wise. The reaction mixture was stirred at room temperature for $2 \mathrm{hrs}$. with constant stirring, the resultant solution was cooled to obtain the crude product. The product was recrystallized from (ethanol/ water) to give the titled compounds.

Synthesis of 3-(1H-benzo[d]imidazol-2-yl)-5-(substituted phenyl) -4,5dihydro isoxazole [21-27], (1H-benzo[d]imidazol-2-yl)-5-(pyridine-3-yl) -4,5- dihydroisoxazole [28] and (3,5-di (1H-benzo [d] imidazol-2-yl) -5methyl-4,5- dihydroisoxazole $[29]^{[18]}$.

To a mixture of $(0.0005 \mathrm{~mol})$ of one of the chalcones [3-11] dissolved in $(10 \mathrm{~mL})$ of dry ethanol, and $(0.0005 \mathrm{~mol}, 0.034 \mathrm{~g})$ hydroxylamine hydrochloride dissolved in ethanol $(10 \mathrm{~mL}), 10 \mathrm{~mL}$ of 10 $\% \mathrm{NaOH}$ were added drop wise. The contents were refluxed for $5 \mathrm{hrs}$. The reaction mixture was then poured into ice cold water, The product was recrystallized from (ethanol) to give the titled compounds.

Synthesis of (3-(1H-benzo[d]imidazol-2-yl)-5-(substitutedphenyl) 4,5 - dihydro-1H-pyrazol-1-yl)(3-nitrophenyl) methanone [30-36], (3(1H-benzo [d]imidazol-2-yl) -5-(pyridine-3-yl) -4,5-dihydro-1H- pyrazol1-yl) (3- nitrophenyl) methanone [37] and (3,5-di(1H-benzo[d]imidazol2-yl) -5-methyl-4,5-dihydro-1H- pyrazol-1-yl)(3- nitrophenyl) methanone $[38]^{[19]}$.

A mixture of $(0.0005 \mathrm{~mol})$ of one of the chalcones [3-11] and $(0.09 \mathrm{~g}, 0.0005 \mathrm{~mol}) 3$-nitrobenzhydrazid dissolved in glacial acetic acid $(20 \mathrm{~mL})$. The contents were refluxed for $10 \mathrm{hrs}$. The reaction mixture was then poured into ice cold water, The product was recrystallized from (ethanol/water) to give the titled compounds.

Synthesis of 3-(1H-benzo[d]imidazol-2-yl)-5-(substitutedphenyl) 4,5 - dihydro -1 H-pyrazol -1- carbothioamide [39-45], (3-(1H-benzo [d] imidazol-2-yl)-5-(pyridine-3-yl) -4,5-dihydro-1H- pyrazol-1- carbothioamide [46] and (3,5-di(1H-benzo[d]imidazol-2-yl) -5-methyl-4,5-dihydro $-1 \mathrm{H}$ - pyrazol-1- carbothioamide) [47] ${ }^{[20]}$.

To a mixture of $(0.001 \mathrm{~mol})$ of one of the chalcones [3-11] in $(10 \mathrm{~mL})$ of dry ethanol, and $(0.0005 \mathrm{~mol}, 0.0455 \mathrm{~g})$ thiosemicarbazide dissolved in ethanol $(10 \mathrm{~mL}), 10 \mathrm{~mL}$ of $10 \% \mathrm{KOH}$ were added drop wise. The contents were reflux temperature for $8 \mathrm{hrs}$. The reaction mixture was then poured into ice cold- water, The product was recrystallized from (ethanol/water) to give the titled compounds.

Synthesis of 3-(1H-benzo[d]imidazol-2-yl)-5-(substituted phenyl) -4,5 - dihydro -1 H-pyrazol -1 - carboxamide [48-54], (3-(1H-benzo [d] imidazol-2-yl)-5-(pyridine-3-yl)-4,5-dihydro-1H- pyrazol-1- carboxamide 
[55] and (3,5-di(1H-benzo[d]imidazol-2-yl) -5-methyl-4,5-dihydro-1Hpyrazol-1- carboxamide) $[56]^{[20]}$.

To a mixture of $(0.0005 \mathrm{~mol})$ of one of the chalcones [3-11] in $(10 \mathrm{~mL})$ of dry ethanol, $(0.0005 \mathrm{~mol}, 0.0375 \mathrm{~g})$ semicarbazide dissolved in ethanol $(10 \mathrm{~mL}), 10 \mathrm{~mL}$ of $10 \% \mathrm{KOH}$ were added drop wise. The contents were refluxed for $8 \mathrm{hrs}$. The reaction mixture was then poured into ice cold- water, The product was recrystallized from (ethanol/water) to give the titled compounds.

Synthesis of १-(1H-benzo[d]imidazol-2-yl)- ₹-(substituted phenyl) -4,5 -dihydropyrimidin-2-amine [57-63], 7-(1H-benzo[d]imidazol-2-yl) 乏-(pyridine-3-yl)-4,5- dihydropyrimidin-2-amine [64] and 4,6-di(1Hbenzo[d]imidazol-2-yl)-5-methyl-4,5-dihydropyrimidin-2-amine) $[65]^{[21]}$.

A mixture of $(0.0005 \mathrm{~mol})$ of one of the chalcones [3-11] in $(10$ $\mathrm{mL})$ of dry ethanol, guanidine nitrate $(0.061 \mathrm{~g}, 0.0005 \mathrm{~mol})$ in $(10 \mathrm{~mL})$ sodium ethoxide were added. The contents were refluxed for $6 \mathrm{hrs}$. The reaction mixture was then cooled, poured into crushed ice and the separated product was filtered, washed with water, dried and recrystallized from ethanol.

Synthesis of 4-(1H-benzo[d]imidazol-2-yl)-2-(substitutedphenyl)2,3- dihydro-1H-benzo[b][1,4] diazepine [66-72], 4-(1H-benzo[d] imidazole -2-yl) -2-(pyridine-3-yl)-2,3- dihydro-1H-benzo [b][1,4] diazepine [73] and 2,4-(1H-benzo[d]imidazol-2-yl) -2-methyl-2,3dihydro-1H-benzo [b] $[1,4]$ diazepine $[74]^{[22]}$.

To a mixture of $(0.0005 \mathrm{~mol})$ of one of the chalcones [3-11] and $(0.054 \mathrm{~g}, 0.0005 \mathrm{~mol})$ ortho phenylenediamine dissolved in dry ethanol $(25 \mathrm{~mL})$ a few drops of glacial acetic acid were added. The contents were refluxed for $10 \mathrm{hrs}$. The reaction mixture was then poured into ice cold water, The product was recrystallized from (ethanol/water) to give the titled compounds.

Synthesis of 4-(1H-benzo[d]imidazol-2-yl)-2-(substituted phenyl) -2,3-dihydrobenzo [b] [1,4] oxazepine [75-81],4-(1H-benzo [d] imidazole -2-yl) -2-(pyridine-3-yl)-2,3- dihydrobenzo [b][1,4] oxazepine [82] and 2,4-(1H-benzo[d]imidazol-2-yl)-2-methyl-2,3- dihydrobenzo [b][1,4] oxazepine $[83]^{[23]}$.

A mixture of $(0.0005 \mathrm{~mol})$ of one of the chalcones [3-11] and $(0.054 \mathrm{~g}, 0.0005 \mathrm{~mol}) 2$-aminophenol dissolved in dry ethanol $(25 \mathrm{~mL})$ and few drops of glacial acetic acid . were refluxed for $10 \mathrm{hrs}$. The reaction mixture was then poured into ice cold water, The product was recrystallized from (ethanol/water) to give the titled compounds. 


\section{RESULTS AND DISCUSSION}

In this paper we investigate the synthesis of heterocyclic compounds [12-83]

From their starting materials, thus ortho phenylen diamine was treated with lactic acid to give 1-(1H-benzo[d]imidazol-2-yl) ethanol [1] in 93\% yield. The IR spectra ${ }^{[24]}$ show absorption at $v_{\mathrm{cm}^{-1}}(3423)(\mathrm{O}-\mathrm{H}), \mathrm{v} \mathrm{cm}^{-1}$ (3171) $(\mathrm{N}-\mathrm{H})$ and $v_{\mathrm{cm}^{-1}}(1621)(\mathrm{C}=\mathrm{N}), \mathrm{U} \cdot \mathrm{V} \lambda \max$ (287) in DMF, ${ }^{1} \mathrm{H}$ NMR [400MHz, $\delta$, ppm, DMSO-d6] 1.503-1.552 $\delta$ (3H,d,CH3), $4.909-4.970 \delta(1 \mathrm{H}, \mathrm{m}, \mathrm{CH}), 5.764-5.776 \delta(1 \mathrm{H}, \mathrm{d}, \mathrm{OH}), 7.096-7.158 \delta$ $(2 \mathrm{H}, \mathrm{m}, \mathrm{Ar}-\mathrm{H}), 7.425-7.553 \delta(2 \mathrm{H}, \mathrm{m}, \mathrm{Ar}-\mathrm{H}), 12.242 \delta(1 \mathrm{H}, \mathrm{S}, \mathrm{N}-\mathrm{H})$, the product is white in colour and has m.p $\left(177-179^{\circ} \mathrm{C}\right)$.

1-(1H-benzo[d]imidazol-2-yl) ethanol was oxidized by Potassium dichromate to give 1-(1H-benzo[d]imidazol-2-yl) ethanone [2] in 70\% yield. The IR spectra ${ }^{[25]}$ show absorption at $\mathrm{v} \mathrm{cm}^{-1}(3290)(\mathrm{N}-\mathrm{H}), \mathrm{v} \mathrm{cm}^{-1}$ (1617) $(\mathrm{C}=\mathrm{N}), \mathrm{v} \mathrm{cm}^{-1}(1675)(\mathrm{C}=\mathrm{O})$, The disappearance of $\mathrm{O}-\mathrm{H}$ band indicate the oxidation of the secondary alcohol to ketone, ${ }^{1} \mathrm{H}$ NMR


$(2 \mathrm{H}, \mathrm{m}, \mathrm{Ar}-\mathrm{H}), 7.693 \delta(2 \mathrm{H}, \mathrm{s}, \mathrm{Ar}-\mathrm{H}), 13.247 \delta(1 \mathrm{H}, \mathrm{S}, \mathrm{N}-\mathrm{H})$ it has Yellow colour of m.p $\left(185-187^{\circ} \mathrm{C}\right)$.

1-(1H-benzo[d]imidazol-2-yl) ethanone was condensed with substituted benzaldehyde through Claisen - Schimidite reaction to give chalcones [3-11]. The IR spectra ${ }^{[26]}$ show absorption bands at $v_{\mathrm{cm}^{-1}}$ $(1654-1674)(\mathrm{C}=\mathrm{O})$ and $v_{\mathrm{cm}^{-1}}(1587-1508)(\mathrm{C}=\mathrm{C}), \lambda_{\max }(281-333) \mathrm{nm}$, (Table 1) ${ }^{1} \mathrm{H}$ NMR [400MHz, $\delta$, ppm, DMSO- $\mathrm{d}_{6}$ ] of Compound[7] give the following signals bands $7.384-7.397 \delta(2 \mathrm{H}, \mathrm{m}, \mathrm{Ar}-\mathrm{H}), 7.462-7.503 \delta$ $(1 \mathrm{H}, \mathrm{t}, \mathrm{di} \quad$ chloroAr-H), $7.635-7.655 \delta \quad(2 \mathrm{H}, \mathrm{d}$, dichloroAr-H), $7.746 \quad \delta$



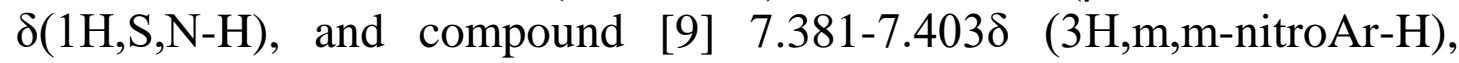
7.767-7.807 $\delta \quad(\alpha 1 \mathrm{H}, \mathrm{d}, \mathrm{CH}), \quad 8.077-8.117 \delta(\beta 1 \mathrm{H}, \mathrm{d}, \mathrm{CH}), \quad 8.260-8.386 \delta$ $(4 \mathrm{H}, \mathrm{m}, \mathrm{Ar}-\mathrm{H}$, benzimidazole), the physical properties and spectral data are listed in Table(1). 


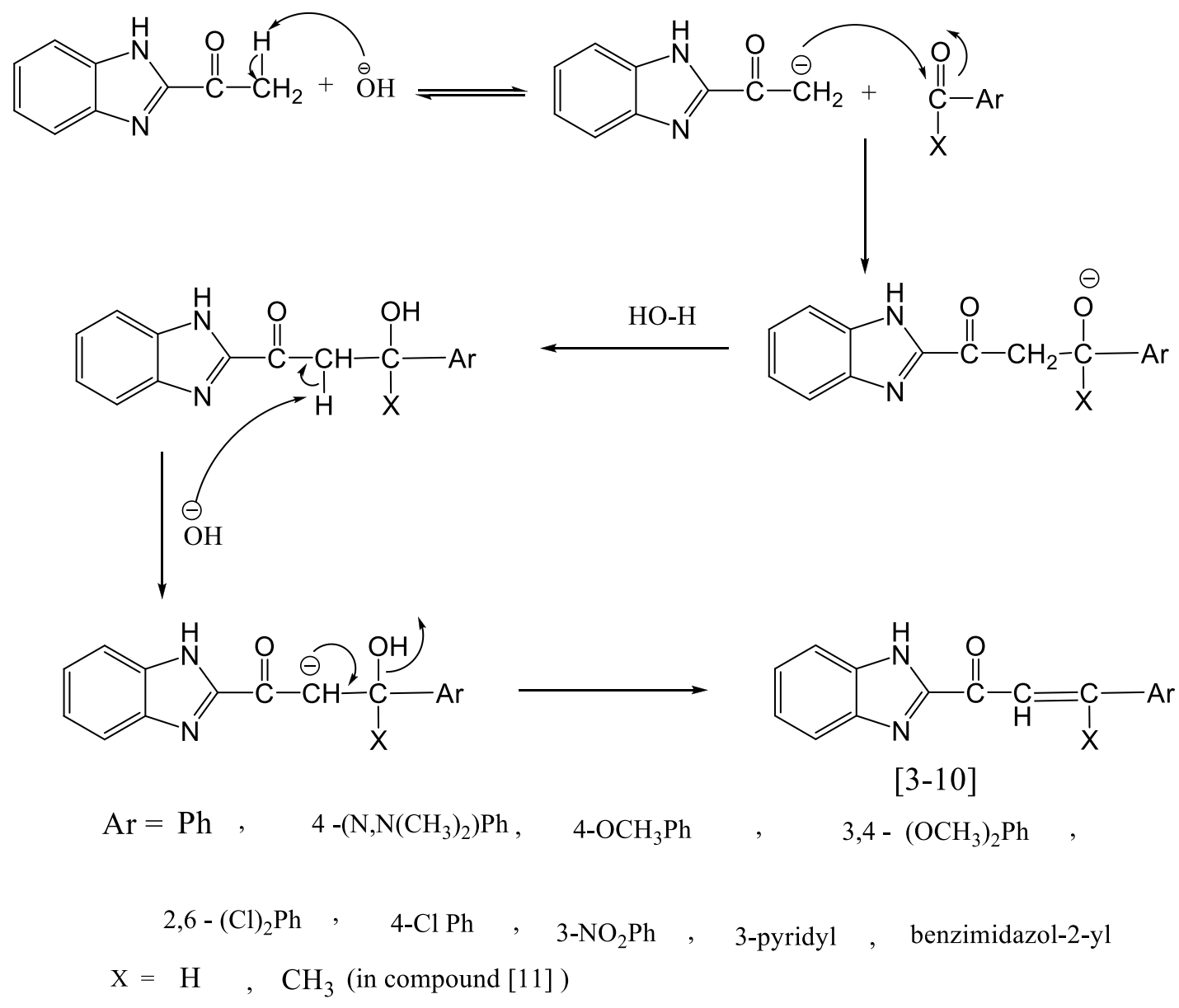

Scheme (1)

The suggested mechanism for the Synthesis of chalcones 
Table(1): Physical properties and spectral data[ $\mathrm{IR}(\mathrm{KBr}) v \mathrm{~cm}^{-1}$ and U.Vnm ]of compounds [3-11]<smiles>[X]C([Al])=CC(=O)c1nc2ccccc2[nH]1</smiles>

\begin{tabular}{|c|c|c|c|c|c|c|c|c|c|}
\hline \multirow{2}{*}{$\begin{array}{c}\text { Com.. } \\
\text { No }\end{array}$} & \multirow[b]{2}{*}{ Ar } & \multirow[b]{2}{*}{$X$} & \multirow[b]{2}{*}{$\begin{array}{l}\text { M.p. } \\
\left({ }^{\circ} \mathrm{C}\right)\end{array}$} & \multirow{2}{*}{$\begin{array}{c}\text { Yiel } \\
\text { d } \\
(\%)\end{array}$} & \multicolumn{4}{|c|}{ IR v cm $\mathrm{cm}^{-1}(\mathrm{KBr})$} & \multirow{2}{*}{$\begin{array}{l}\text { U.V } \\
\text { inDMF } \\
\lambda_{\max }, \\
(\mathrm{nm})\end{array}$} \\
\hline & & & & & $\mathrm{C}=\mathrm{O}$ & N-H & $\begin{array}{c}\mathrm{C}-\mathrm{H} \\
\mathrm{Ar}\end{array}$ & $\mathrm{C}=\mathrm{C}$ & \\
\hline 3 & $\mathrm{Ph}$ & $\mathrm{H}$ & $\begin{array}{l}199- \\
203\end{array}$ & 88 & 1662 & 3251 & 3063 & 1573 & 325 \\
\hline 4 & $4-\mathrm{N}, \mathrm{N}\left(\mathrm{CH}_{3}\right)_{2} \mathrm{Ph}$ & $\mathrm{H}$ & $\begin{array}{l}240- \\
242\end{array}$ & 60 & 1660 & 3282 & 3066 & 1561 & 211 \\
\hline 5 & $4-\mathrm{OCH}_{3} \mathrm{Ph}$ & $\mathrm{H}$ & $\begin{array}{l}180- \\
182\end{array}$ & 89 & 1654 & 3260 & 3064 & 1576 & 326 \\
\hline 6 & $3,4\left(\mathrm{OCH}_{3}\right)_{2} \mathrm{Ph}$ & $\mathrm{H}$ & $\begin{array}{l}204- \\
206\end{array}$ & 77 & 1654 & 3247 & 3015 & 1587 & 251 \\
\hline 7 & $2,6(\mathrm{Cl})_{2} \mathrm{Ph}$ & $\mathrm{H}$ & $\begin{array}{l}197- \\
198\end{array}$ & 81 & 1659 & 3279 & 3069 & 1553 & 266 \\
\hline 8 & 4-Cl Ph & $\mathrm{H}$ & $\begin{array}{l}208- \\
210\end{array}$ & 92 & 1665 & 3559 & 3967 & 1566 & 372 \\
\hline 9 & $3-\mathrm{NO}_{2} \mathrm{Ph}$ & $\mathrm{H}$ & $\begin{array}{l}218- \\
220\end{array}$ & 93 & 1666 & 3417 & 3031 & 1569 & 287 \\
\hline 10 & 3-pyridyl & $\mathrm{H}$ & $\begin{array}{l}209- \\
211\end{array}$ & 90 & 1668 & 3422 & 3158 & 1585 & 326 \\
\hline 11 & $\begin{array}{c}\text { Benzimidazol } \\
-2-y l\end{array}$ & $\mathrm{CH}_{3}$ & $\begin{array}{l}169- \\
171\end{array}$ & 75 & 1674 & 3289 & 3059 & 1508 & 310 \\
\hline
\end{tabular}

The chalcones were treated with hydrogen peroxide in ethanol in the presence of sodium hydroxide to give the oxiran compound[12-20]. The IR spectra ${ }^{(27]}$ of compounds [12-20] show absorption bands at $v_{\mathrm{cm}^{-1}}$ $(1675-1734) \quad(\mathrm{C}=\mathrm{O}) \quad$ and $\mathrm{v} \mathrm{cm}^{-1}(1581-1662)(\mathrm{C}=\mathrm{N}), \lambda \neg \max (347-275$ $\mathrm{nm}$ )and (269-236 nm), (Table2). ${ }^{1} \mathrm{H}$ NMR [400MHz, $\delta$, ppm, DMSO-d ${ }_{6}$ ] of compound[14], $3.390 \delta(1 \mathrm{H}, \mathrm{S}, \mathrm{COCH}$-epoxy ), $3.825 \delta(3 \mathrm{H}, \mathrm{S},-\mathrm{OCH} 3)$, $7.010-7.031 \delta \quad 2 \mathrm{H}, \mathrm{d}, \mathrm{Ar}-\mathrm{H}), 7.181-7.203 \delta(2 \mathrm{H}, \mathrm{m}, \mathrm{Ar}-\mathrm{H}), 7.584-7.606 \delta$




$12.609 \delta(1 \mathrm{H}, \mathrm{S}, \mathrm{N}-\mathrm{H})$, the physical properties and spectral data are listed in Table (2).

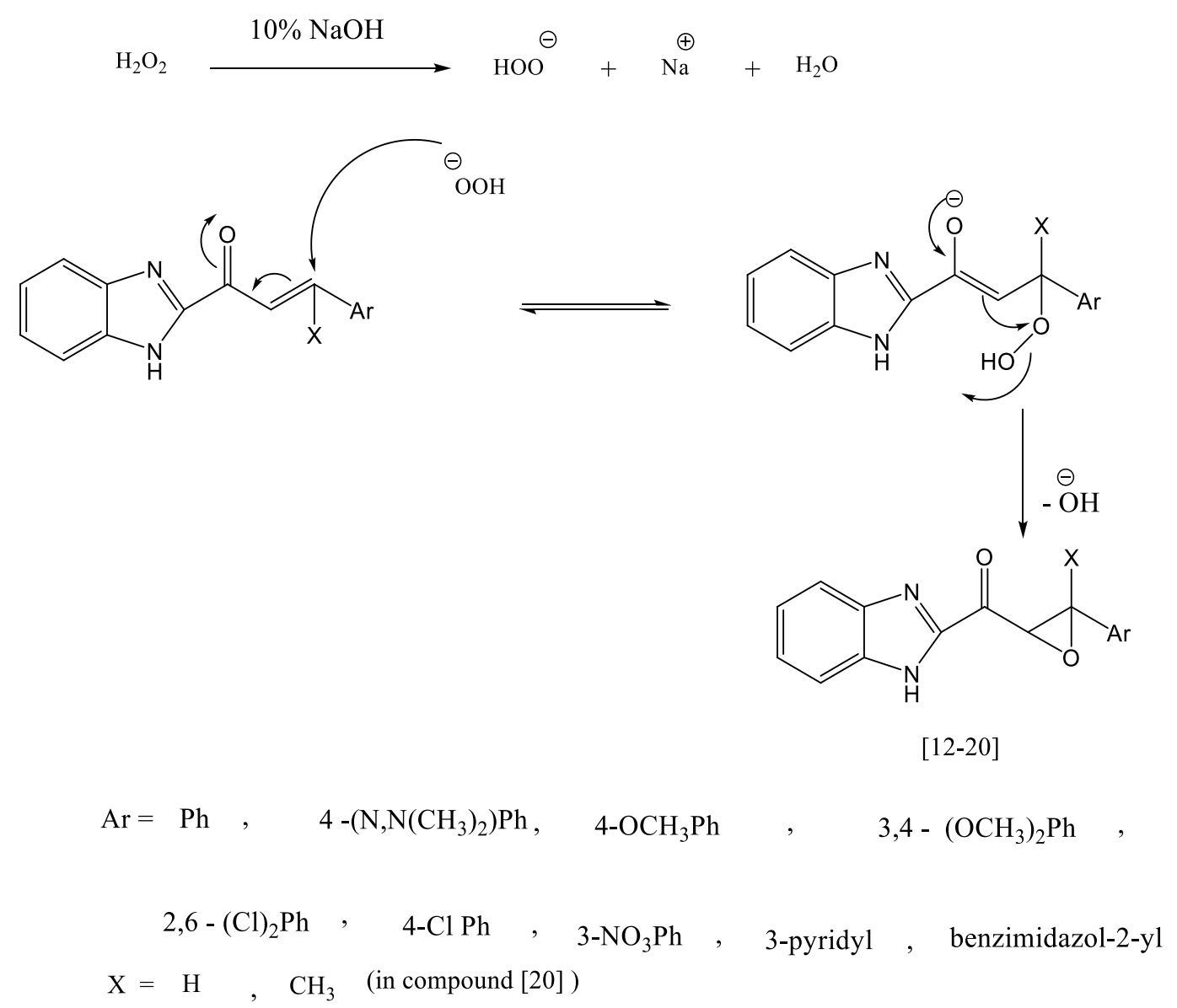

Scheme (2)

The suggested mechanism for the reaction of chalcones with hydrogen peroxide 
Table(2): Physical properties and spectral data [IR (KBr) $v \mathrm{~cm}^{-1}$ and U.Vnm] of compounds [12-20]<smiles>[X]C1(Br)OC1C(=O)c1nc2ccccc2[nH]1</smiles>

\begin{tabular}{|c|c|c|c|c|c|c|c|c|c|c|}
\hline \multirow{2}{*}{$\begin{array}{c}\text { Comp. } \\
\text { No }\end{array}$} & \multirow{2}{*}{$\mathrm{Ar}$} & \multirow{2}{*}{ X } & \multirow{2}{*}{$\begin{array}{l}\text { M.p. } \\
\left({ }^{\circ} \mathrm{C}\right)\end{array}$} & \multirow{2}{*}{$\begin{array}{r}\text { Yiel } \\
\mathrm{d}(\%)\end{array}$} & \multicolumn{5}{|c|}{ IR $v \mathrm{~cm}^{-1}(\mathrm{KBr})$} & \multirow{2}{*}{$\begin{array}{c}\text { U.V } \\
\text { In } \\
\text { DMF } \\
\lambda_{\max }, \\
(\mathrm{nm}) \\
\end{array}$} \\
\hline & & & & & $\mathrm{C}=\mathrm{O}$ & $\mathrm{N}-\mathrm{H}$ & $\mathrm{C}=\mathrm{N}$ & $c \stackrel{C}{C}$ & $\mathrm{C}-\mathrm{N}$ & \\
\hline Ir & $\mathrm{Ph}$ & $\mathrm{H}$ & $\begin{array}{l}280 \\
\text { dec. }\end{array}$ & 62 & $16 \wedge r$ & $r \leqslant \varepsilon r$ & 1646 & $\begin{array}{l}\text { as } 1106 \\
\text { sy } 1 \cdot r \text { r }\end{array}$ & 1253 & $\begin{array}{l}\text { rat } \\
\text { rTV }\end{array}$ \\
\hline ir & $\varepsilon-\mathrm{N}, \mathrm{N}\left(\mathrm{CH}_{3}\right)_{2} \mathrm{Ph}$ & $\mathrm{H}$ & $\begin{array}{l}168 \\
\text { dec. }\end{array}$ & 73 & $1 \vee \cdot 0$ & TMt & 1662 & $\begin{array}{l}\text { as } 1190 \\
\text { sy } 1.74\end{array}$ & 1230 & $\begin{array}{l}\text { r.q } \\
\text { ror }\end{array}$ \\
\hline $1 \varepsilon$ & 4- $\mathrm{OCH}_{3} \mathrm{Ph}$ & $\mathrm{H}$ & $\begin{array}{c}155- \\
156\end{array}$ & 63 & 1610 & $r \leqslant r$ & 1655 & $\begin{array}{l}\text { as } 1180 \\
\text { sy } 1 \cdot \text { r }\end{array}$ & 1228 & $\begin{array}{l}\text { tru } \\
\text { tru }\end{array}$ \\
\hline 10 & $3,4-\left(\mathrm{OCH}_{3}\right)_{2} \mathrm{Ph}$ & $\mathrm{H}$ & $\begin{array}{l}210 \\
\text { dec. }\end{array}$ & 68 & $1 \vee \mu \varepsilon$ & $r \varepsilon \cdot \varepsilon$ & 1647 & $\begin{array}{l}\text { as } 1107 \\
\text { syl.rr }\end{array}$ & (13r & $\begin{array}{l}r V O \\
r \leqslant T\end{array}$ \\
\hline 17 & $2,6-(\mathrm{Cl})_{2} \mathrm{Ph}$ & $\mathrm{H}$ & $\begin{array}{l}178 \\
\text { dec. }\end{array}$ & 63 & $16 \mathrm{Vo}$ & rrql & 1581 & $\begin{array}{l}\text { as } 1140 \\
\text { sy } 1 \cdot \wedge v\end{array}$ & 1217 & $\begin{array}{l}r \leqslant V \\
r 7 q\end{array}$ \\
\hline IV & 4- $\mathrm{Cl} \mathrm{Ph}$ & $\mathrm{H}$ & $\begin{array}{l}162- \\
163\end{array}$ & 75 & 1694 & $r \leqslant 90$ & 1620 & $\begin{array}{l}\text { as } 1176 \\
\text { sy } 1.89\end{array}$ & 1228 & 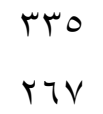 \\
\hline 11 & $3-\mathrm{NO}_{2} \mathrm{Ph}$ & $\mathrm{H}$ & $\begin{array}{l}174- \\
177\end{array}$ & 82 & $16 \wedge 9$ & $r \varepsilon \cdot \wedge$ & 1620 & $\begin{array}{l}\text { as } 1141 \\
\text { sy } 1099\end{array}$ & 1312 & \\
\hline 19 & 3-pyridyl & $\mathrm{H}$ & $\begin{array}{l}270 \\
\text { dec. }\end{array}$ & 70 & $16 \wedge \varepsilon$ & $r \leqslant r)$ & 1661 & $\begin{array}{l}\text { as } 1093 \\
\text { sy } 90 \wedge\end{array}$ & 1317 & $\begin{array}{l}r .0 \\
r \leqslant r\end{array}$ \\
\hline r. & $\begin{array}{c}\text { Benzimidazol- } \\
2-y l\end{array}$ & $\mathrm{CH}_{3}$ & $\begin{array}{c}178- \\
180\end{array}$ & 75 & $16 \wedge \vee$ & $32 \vee 9$ & 1611 & $\begin{array}{l}\text { as } 1147 \\
\text { sy } 1007\end{array}$ & 1241 & \\
\hline
\end{tabular}

The chalcones was treated with hydroxylamine hydrochloride in the presence of sodium hydroxide to give the isoxazoline compounds[2129] The IR spectra of compounds [21-29] show absorption at (930-995) $v \mathrm{~cm}^{-1}(\mathrm{~N}-\mathrm{O})$ and $(1609-1664) v \mathrm{~cm}^{-1}(\mathrm{C}=\mathrm{N})^{[28]}, \lambda \neg \max (298-262 \mathrm{~nm})$ 
and (352-301 nm), (Table3). ${ }^{1} \mathrm{HNMR}$ [400MHz, $\delta, \mathrm{ppm}$, DMSO-d ${ }^{6}$ ]. compounds[23] give the following signals $3.704 \delta$ (3H,S,-OCH3), $3.748-3.806 \delta(1 \mathrm{H}, \mathrm{t},-\mathrm{CH}), \quad 3.850-3.859 \delta\left(2 \mathrm{H}, \mathrm{d},-\mathrm{CH}_{2}\right), \quad 7.135-7.203 \delta$ $(2 \mathrm{H}, \mathrm{t}, \mathrm{Ar}-\mathrm{H}) \delta, 7.419-7.439 \delta(2 \mathrm{H}, \mathrm{d}, \mathrm{Ar}-\mathrm{H}), 7.518-7.538 \delta \quad(2 \mathrm{H}, \mathrm{d}, \mathrm{Ar}-\mathrm{H})$, $7.935-7.975 \delta(2 \mathrm{H}, \mathrm{t}, \mathrm{Ar}-\mathrm{H}), 11.696 \delta(1 \mathrm{H}, \mathrm{S}, \mathrm{N}-\mathrm{H})$, the physical properties and spectral data are listed in Table (3).



Scheme (3)

The suggested mechanism for the reaction of chalcones with hydroxylamine hydrochloride 
Table(3): Physical properties and spectral data [IR (KBr) $v \mathrm{~cm}^{-1}$ and U.Vnm] of compounds [21-29]

\begin{tabular}{|c|c|c|c|c|c|c|c|c|c|}
\hline \multirow{2}{*}{$\begin{array}{c}\text { Comp. } \\
\text { No }\end{array}$} & \multirow{2}{*}{$\mathrm{Ar}$} & \multirow{2}{*}{$X$} & \multirow{2}{*}{$\begin{array}{l}\text { M.p. } \\
\left({ }^{\circ} \mathrm{C}\right)\end{array}$} & \multirow{2}{*}{$\begin{array}{c}\text { Yiel } \\
\mathrm{d}(\%)\end{array}$} & \multicolumn{4}{|c|}{ IR $v \mathrm{~cm}^{-1}(\mathrm{KBr})$} & \multirow{2}{*}{$\begin{array}{c}\text { U.V } \\
\text { inDMF } \\
\lambda_{\max }, \\
(\mathrm{nm})\end{array}$} \\
\hline & & & & & $\mathrm{C}=\mathrm{N}$ & $\mathrm{C}=\mathrm{C}$ & $\mathrm{N}-\mathrm{H}$ & $\mathrm{N}-\mathrm{O}$ & \\
\hline 21 & $\mathrm{Ph}$ & $\mathrm{H}$ & $\begin{array}{c}95- \\
97\end{array}$ & 80 & 1619 & 1596 & 3396 & 968 & $\begin{array}{l}329 \\
284\end{array}$ \\
\hline 22 & $\varepsilon-\mathrm{N}, \mathrm{N}\left(\mathrm{CH}_{3}\right)_{2} \mathrm{Ph}$ & $\mathrm{H}$ & $\begin{array}{c}130- \\
133\end{array}$ & 71 & 1610 & 1521 & 3216 & 943 & $\begin{array}{l}338 \\
292\end{array}$ \\
\hline 23 & $4-\mathrm{OCH}_{3} \mathrm{Ph}$ & $\mathrm{H}$ & $\begin{array}{c}104- \\
106\end{array}$ & 69 & 1609 & 1542 & 3221 & 965 & $\begin{array}{l}317 \\
298\end{array}$ \\
\hline 24 & $3,4-\left(\mathrm{OCH}_{3}\right)_{2} \mathrm{Ph}$ & $\mathrm{H}$ & $\begin{array}{c}153- \\
156\end{array}$ & 75 & 1611 & 1557 & 3419 & 971 & $\begin{array}{l}339 \\
285\end{array}$ \\
\hline 25 & $2,6-(\mathrm{Cl})_{2} \mathrm{Ph}$ & $\mathrm{H}$ & $\begin{array}{l}250 \\
\text { dec. }\end{array}$ & 66 & 1664 & 1562 & 3242 & 980 & $\begin{array}{l}304 \\
262\end{array}$ \\
\hline 26 & 4-Cl Ph & $\mathrm{H}$ & $\begin{array}{l}205- \\
207\end{array}$ & 78 & 1614 & 1594 & 3178 & 968 & $\begin{array}{l}352 \\
299\end{array}$ \\
\hline 27 & $3-\mathrm{NO}_{2} \mathrm{Ph}$ & $\mathrm{H}$ & $\begin{array}{l}203 \\
\text { dec. }\end{array}$ & 84 & 1628 & 1558 & 3363 & 930 & $\begin{array}{l}311 \\
266\end{array}$ \\
\hline 28 & 3-pyridyl & $\mathrm{H}$ & $\begin{array}{l}265 \\
\text { dec. }\end{array}$ & 79 & 1655 & 1610 & 3240 & 984 & $\begin{array}{l}325 \\
294\end{array}$ \\
\hline 29 & Benzimidazol-2-yl & $\mathrm{CH}_{3}$ & $\begin{array}{l}234- \\
237\end{array}$ & 65 & 1662 & 1652 & 3375 & 995 & $\begin{array}{l}301 \\
270\end{array}$ \\
\hline
\end{tabular}

The chalcones were treated with 3-nitrobenzohydrazide in the presence of glacial acetic acid to give the pyrazoline compounds [30-38]. The IR spectra of compounds [30-38] show absorption at(1638-1697) $v$ $\mathrm{cm}^{-1} \quad(\mathrm{C}=\mathrm{O})^{[29]}$ amide and(1593-1664) $v \mathrm{~cm}^{-1}(\mathrm{C}=\mathrm{N}), \lambda \neg \max (362-$ $268 \mathrm{~nm})$ and $(267-204 \mathrm{~nm})$, (Table4). ${ }^{1} \mathrm{HNMR}$ [400MHz, $\delta$, ppm, DMSO$\left.\mathrm{d}^{6}\right]$ of compound [32], $3.350 \delta(3 \mathrm{H}, \mathrm{S},-\mathrm{OCH} 3), 3.848 \delta\left(2 \mathrm{H}, \mathrm{d},-\mathrm{CH}_{2}\right)$, 3.887-3. $918 \boldsymbol{\delta}(1 \mathrm{H}, \mathrm{t},-\mathrm{CH}), 7.052-7.074 \delta(2 \mathrm{H}, \mathrm{d}, \mathrm{Ar}-\mathrm{H}), 7.578-7.598 \delta$ (2H,d,Ar-H), $7.316-7.427 \delta(3 \mathrm{H}, \mathrm{m}, \mathrm{m}-$ nitro Ar-H), $7.987 \delta(1 \mathrm{H}, \mathrm{S}, \mathrm{m}-$ nitro $\quad$ Ar-H) $7.849 \quad-7.863 \delta \quad(2 \mathrm{H}, \mathrm{d}, \mathrm{Ar}-\mathrm{H}), 7.871-7.882 \delta \quad(2 \mathrm{H}, \mathrm{d}, \mathrm{Ar}-\mathrm{H})$, $13.456 \delta(1 \mathrm{H}, \mathrm{S}, \mathrm{N}-\mathrm{H})$, the physical properties and spectral data are listed in Table (4). 




[30-38]

$$
\begin{aligned}
\mathrm{Ar}= & \mathrm{Ph}, \quad 4-\left(\mathrm{N}, \mathrm{N}\left(\mathrm{CH}_{3}\right)_{2}\right) \mathrm{Ph}, \quad 4-\mathrm{OCH}_{3} \mathrm{Ph}, \quad 3,4-\left(\mathrm{OCH}_{3}\right)_{2} \mathrm{Ph}, \\
& 2,6-(\mathrm{Cl})_{2} \mathrm{Ph}, \quad 4-\mathrm{Cl} \mathrm{Ph}, \quad 3-\mathrm{NO}_{3} \mathrm{Ph} \quad, \quad 3 \text {-pyridyl }, \text { benzimidazol-2-yl } \\
\mathrm{X}= & \left.\mathrm{H} \quad, \quad \mathrm{CH}_{3} \quad \text { (in compound [47] }\right)
\end{aligned}
$$

Scheme (4)

The suggested mechanism for the reaction of chalcones with 3-nitrobenzohydrazide 
Table(4): Physical properties and spectral data $\left[\mathrm{IR}(\mathrm{KBr}) v \mathrm{~cm}^{-1}\right.$ and U.Vnm] of compounds [30-38]
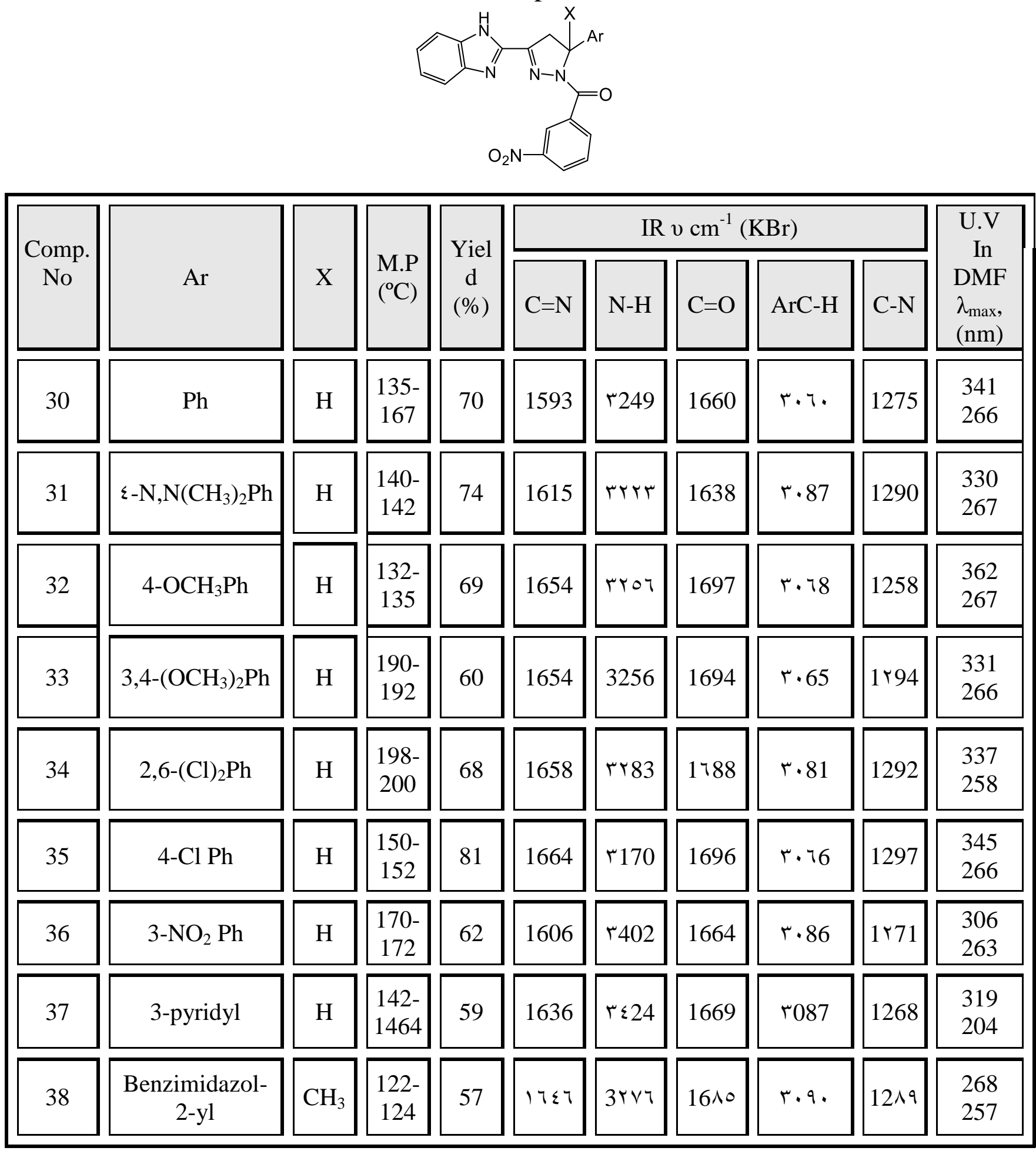

The chalcones was treated with thiosemicarbazide in the presence of potassium hydroxide to give the pyrazoline compound[39-47]. The IR spectra of compounds [39-47] show absorption at (1105-1227) $\mathrm{v} \mathrm{cm}^{-1}$ $(\mathrm{C}=\mathrm{S})^{[30]}$ and (1594-1625)v cm $\mathrm{c}^{-1}(\mathrm{C}=\mathrm{N}), \lambda \neg \max (369-310 \mathrm{~nm})$ and(327265nm).(Table5). ${ }^{1} \mathrm{HNMR} \quad\left[400 \mathrm{MHz}, \delta, \quad\right.$ ppm, DMSO-d $\left.{ }^{6}\right]$ of 
compound[๕0], $3.361 \delta \quad\left(3 \mathrm{H}, \mathrm{S},-\mathrm{N}(\mathrm{CH} 3)_{2}\right), 6.687-6.709 \delta\left(1 \mathrm{H}, \mathrm{d},-\mathrm{CH}_{2}\right)$, $7.191-7.291 \delta(2 \mathrm{H}, \mathrm{t},-\mathrm{CH}), \quad 7.488 \quad-7.508 \quad \boldsymbol{\delta}(2 \mathrm{H}, \mathrm{d}, \mathrm{Ar}-\mathrm{H}), 7.667-7.687 \quad \delta$ (2H,t,Ar-H), $7.571-7.593 \delta \quad(2 \mathrm{H}, \mathrm{d}, \mathrm{Ar}-\mathrm{H}), 7.776 \delta(2 \mathrm{H}, \mathrm{s}, \mathrm{Ar}-\mathrm{H}), 7.932 \delta$ $(2 \mathrm{H}, \mathrm{s}, \mathrm{Ar}-\mathrm{H}), 8.016 \delta(2 \mathrm{H}, \mathrm{s}, \mathrm{Ar}-\mathrm{H}), 8.559-8.606 \delta(1 \mathrm{H}, \mathrm{d}, \mathrm{NH} 2), 13.766 \delta$ $(1 \mathrm{H}, \mathrm{S}, \mathrm{N}-\mathrm{H})$, the physical properties and spectral data are listed in Table (5).

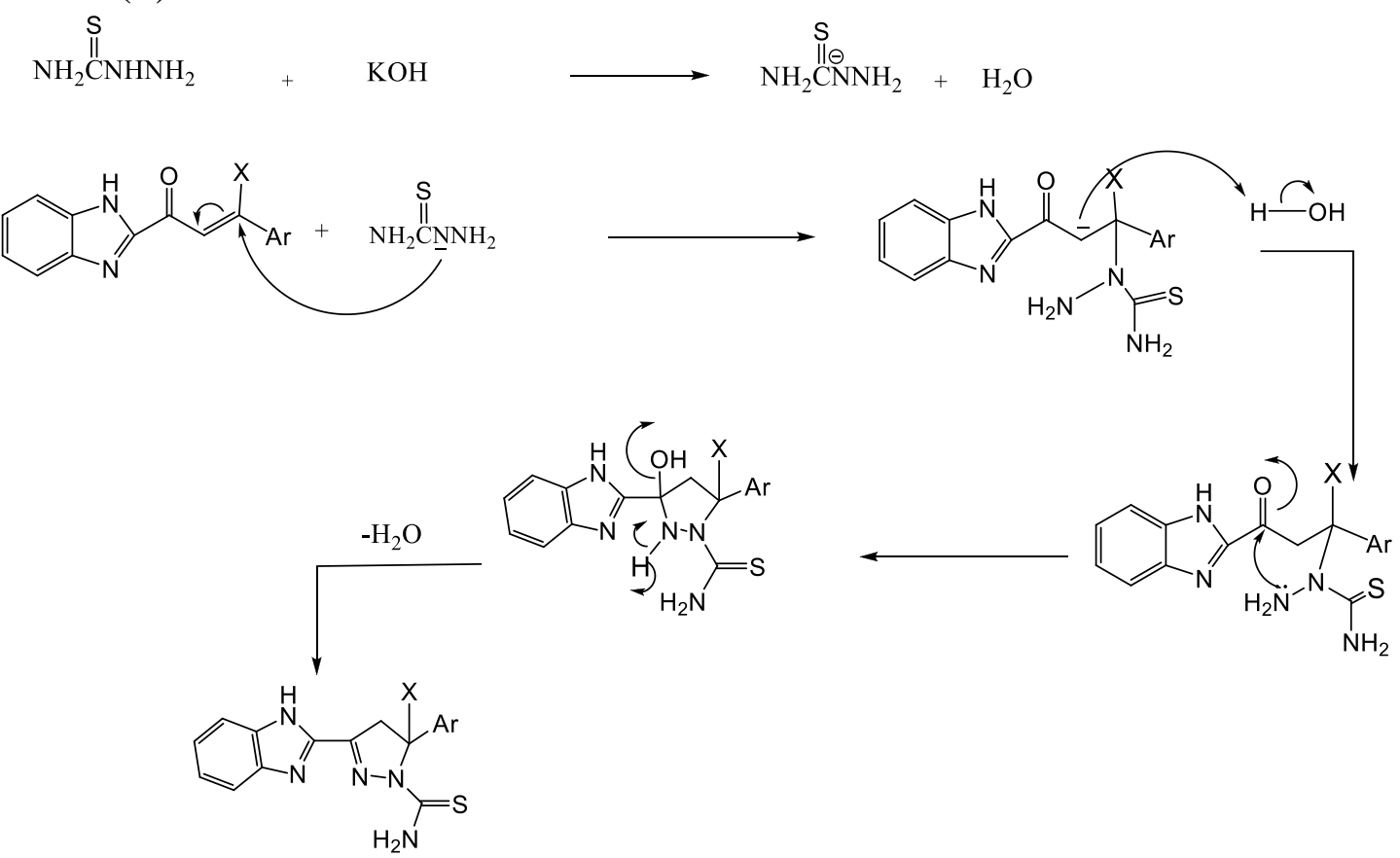

[39-47]

$$
\begin{aligned}
\mathrm{Ar}= & \mathrm{Ph}, \quad 4-\left(\mathrm{N}, \mathrm{N}\left(\mathrm{CH}_{3}\right)_{2}\right) \mathrm{Ph}, \quad 4-\mathrm{OCH}_{3} \mathrm{Ph}, \quad 3,4-\left(\mathrm{OCH}_{3}\right)_{2} \mathrm{Ph}, \\
& 2,6-(\mathrm{Cl})_{2} \mathrm{Ph}, \quad 4-\mathrm{Cl} \mathrm{Ph}, \quad 3-\mathrm{NO}_{3} \mathrm{Ph}, \quad 3 \text {-pyridyl , benzimidazol-2-yl } \\
\mathrm{X}= & \mathrm{H} \quad, \quad \mathrm{CH}_{3} \text { (in compound [56]) }
\end{aligned}
$$

Scheme (5)

The suggested mechanism for the reaction of chalcones with Thiosemicarbazide 
Table(5): Physical properties and spectral data [IR (KBr) $v \mathrm{~cm}^{-1}$ and U.Vnm] of compounds [39-47]<smiles>[X]C1(Br)CC(c2nc3ccccc3[nH]2)=NN1C(N)=S</smiles>

\begin{tabular}{|c|c|c|c|c|c|c|c|c|c|c|}
\hline \multirow{2}{*}{$\begin{array}{c}\text { Comp. } \\
\text { No }\end{array}$} & \multirow[b]{2}{*}{$\mathrm{Ar}$} & \multirow[b]{2}{*}{$X$} & \multirow{2}{*}{$\begin{array}{l}\text { M.P } \\
\left({ }^{\circ} \mathrm{C}\right)\end{array}$} & \multirow{2}{*}{$\begin{array}{c}\text { Yiel } \\
\mathrm{d} \\
(\%)\end{array}$} & \multicolumn{5}{|c|}{ IR $v \mathrm{~cm}^{-1}(\mathrm{KBr})$} & \multirow{2}{*}{$\begin{array}{c}\text { U.V } \\
\text { In } \\
\text { DMF } \\
\lambda_{\max }, \\
(\mathrm{nm})\end{array}$} \\
\hline & & & & & $\mathrm{C}=\mathrm{S}$ & $\mathrm{C}-\mathrm{N}$ & $\mathrm{C}=\mathrm{N}$ & $\mathrm{N}-\mathrm{N}$ & $\mathrm{NH}_{2}$ & \\
\hline 39 & $\mathrm{Ph}$ & $\mathrm{H}$ & \begin{tabular}{|c|}
$197-$ \\
199
\end{tabular} & 52 & 1227 & 1276 & 1598 & 1093 & $\begin{array}{l}3420 \\
3260\end{array}$ & $\begin{array}{l}362 \\
276\end{array}$ \\
\hline 40 & $\varepsilon-\mathrm{N}, \mathrm{N}\left(\mathrm{CH}_{3}\right)_{2} \mathrm{Ph}$ & $\mathrm{H}$ & $\begin{array}{l}165- \\
167\end{array}$ & 79 & 1183 & 1280 & 1606 & 1058 & $\begin{array}{l}3416 \\
3257\end{array}$ & $\begin{array}{l}327 \\
276\end{array}$ \\
\hline 41 & $4-\mathrm{OCH}_{3} \mathrm{Ph}$ & $\mathrm{H}$ & $\begin{array}{c}108- \\
110\end{array}$ & 71 & 1169 & 1249 & 1604 & 1026 & $\begin{array}{l}3454 \\
3330\end{array}$ & $\begin{array}{l}310 \\
278\end{array}$ \\
\hline 42 & $3,4-\left(\mathrm{OCH}_{3}\right)_{2} \mathrm{Ph}$ & $\mathrm{H}$ & $\begin{array}{c}138- \\
140\end{array}$ & 57 & 1163 & 1265 & 1601 & 1032 & $\begin{array}{l}3423 \\
3281\end{array}$ & $\begin{array}{l}369 \\
327\end{array}$ \\
\hline 43 & $2,6-(\mathrm{Cl})_{2} \mathrm{Ph}$ & $\mathrm{H}$ & $\begin{array}{c}208- \\
210\end{array}$ & 76 & 1192 & 1275 & 1594 & 1071 & $\begin{array}{l}3415 \\
3251\end{array}$ & $\begin{array}{l}358 \\
304\end{array}$ \\
\hline 44 & 4-Cl Ph & $\mathrm{H}$ & $\begin{array}{c}177- \\
179\end{array}$ & 75 & 1176 & 1283 & 1601 & 1091 & $\begin{array}{l}3438 \\
3280\end{array}$ & $\begin{array}{l}357 \\
279\end{array}$ \\
\hline 45 & $3-\mathrm{NO}_{2} \mathrm{Ph}$ & $\mathrm{H}$ & $\begin{array}{c}145- \\
148\end{array}$ & 77 & 1144 & 1277 & 1618 & 1097 & 3417 & $\begin{array}{l}317 \\
278\end{array}$ \\
\hline 46 & 3-pyridyl & $\mathrm{H}$ & $\begin{array}{c}187- \\
190\end{array}$ & 75 & 1113 & 1278 & 1625 & 1047 & $\begin{array}{l}3440 \\
\text { 3ro. }\end{array}$ & $\begin{array}{l}282 \\
265\end{array}$ \\
\hline 47 & $\begin{array}{c}\text { Benzimidazol- } \\
2-y l\end{array}$ & $\mathrm{CH}_{3}$ & $\begin{array}{l}215- \\
217\end{array}$ & 52 & 1105 & 1276 & 1609 & 1040 & $\begin{array}{l}3415 \\
3272\end{array}$ & $\begin{array}{l}358 \\
266\end{array}$ \\
\hline
\end{tabular}

The chalcones was treated with semicarbazide in the presence of potassium hydroxide to give the pyrazoline compound[48-56]. The IR spectra of compounds [48-56] show absorption at (1661-1713) $\mathrm{v} \mathrm{cm}^{-1}$ $(\mathrm{C}=\mathrm{O})^{[31]}$ amide and(1575-1669)v $\mathrm{cm}^{-1}(\mathrm{C}=\mathrm{N}), \lambda \neg \max \quad(211-326) \mathrm{nm}$. (Table 6). ${ }^{1} \mathrm{HNMR}$ [400MHz, $\delta, \mathrm{ppm}$, DMSO-d $\left.{ }^{6}\right]$ of compounds[52] $3.346 \delta(2 \mathrm{H}, \mathrm{d},-\mathrm{CH} 2), 7.468-7.527 \delta(2 \mathrm{H}, \mathrm{d}$, di chloroAr-H), $8.202-8.322 \delta$ $(1 \mathrm{H}, \mathrm{t}, \mathrm{di} \quad$ chloroAr-H), $7.640 \quad-7.660 \quad \delta(2 \mathrm{H}, \mathrm{d}, \mathrm{Ar}-\mathrm{H}), \quad \delta \quad 7.875 \quad-7.895$ $(2 \mathrm{H}, \mathrm{d}, \mathrm{Ar}-\mathrm{H}), 8.202-8.322 \delta(1 \mathrm{H}, \mathrm{t},-\mathrm{CH}), 11.774 \delta\left(1 \mathrm{H}, \mathrm{S}, \mathrm{NH}_{2}\right), 13.626 \delta$ 
$(1 \mathrm{H}, \mathrm{S}, \mathrm{N}-\mathrm{H})$, the physical properties and spectral data are listed in Table (6).

Table(6): Physical properties and spectral data [IR $(\mathrm{KBr}) \mathrm{v} \mathrm{cm}^{-1}$ and U.Vnm] of compounds [48-56]<smiles>[X]C1([Al])CC(c2nc3ccccc3[nH]2)=NN1C(N)=O</smiles>

\begin{tabular}{|c|c|c|c|c|c|c|c|c|c|c|}
\hline \multirow{2}{*}{$\begin{array}{c}\text { Comp. } \\
\text { No }\end{array}$} & \multirow[b]{2}{*}{$\mathrm{Ar}$} & \multirow[b]{2}{*}{$X$} & \multirow{2}{*}{$\begin{array}{l}\text { M.P } \\
\left({ }^{\circ} \mathrm{C}\right)\end{array}$} & \multirow{2}{*}{$\begin{array}{c}\text { Yiel } \\
\mathrm{d} \\
(\%)\end{array}$} & \multicolumn{5}{|c|}{ IR $v \mathrm{~cm}^{-1}(\mathrm{KBr})$} & \multirow{2}{*}{$\begin{array}{c}\mathrm{U.V} \\
\text { In } \\
\mathrm{DMF} \\
\lambda_{\max }, \\
(\mathrm{nm}) \\
\end{array}$} \\
\hline & & & & & $\mathrm{C}=\mathrm{O}$ & $\mathrm{C}-\mathrm{N}$ & $\mathrm{C}=\mathrm{N}$ & $\mathrm{N}-\mathrm{N}$ & $\mathrm{NH}_{2}$ & \\
\hline 48 & $\mathrm{Ph}$ & $\mathrm{H}$ & $\begin{array}{c}189- \\
200\end{array}$ & 80 & 1684 & 1288 & 1647 & 1092 & $\begin{array}{l}3462 \\
3290\end{array}$ & $\begin{array}{l}330 \\
289\end{array}$ \\
\hline 49 & $\varepsilon-\mathrm{N}, \mathrm{N}\left(\mathrm{CH}_{3}\right)_{2} \mathrm{Ph}$ & $\mathrm{H}$ & $\begin{array}{l}230 \\
\text { dec. }\end{array}$ & 72 & 1684 & 1229 & 1609 & 1013 & 3460 & $\begin{array}{l}317 \\
262\end{array}$ \\
\hline 50 & $4-\mathrm{OCH}_{3} \mathrm{Ph}$ & $\mathrm{H}$ & $\begin{array}{l}270 \\
\text { dec. }\end{array}$ & 67 & $16 \wedge 9$ & 1252 & 1646 & 1088 & $\begin{array}{l}3454 \\
\text { r } 82\end{array}$ & $\begin{array}{l}314 \\
298\end{array}$ \\
\hline 51 & $3,4-\left(\mathrm{OCH}_{3}\right)_{2} \mathrm{Ph}$ & $\mathrm{H}$ & $\begin{array}{l}189- \\
191\end{array}$ & 78 & 1664 & 1238 & 1624 & 1081 & 3433 & $\begin{array}{l}336 \\
263\end{array}$ \\
\hline 52 & $2,6-(\mathrm{Cl})_{2} \mathrm{Ph}$ & $\mathrm{H}$ & $\begin{array}{l}200- \\
202\end{array}$ & 57 & 1661 & 1213 & 1594 & 1072 & $\begin{array}{l}3415 \\
3281\end{array}$ & $\begin{array}{l}321 \\
264\end{array}$ \\
\hline 53 & 4-Cl Ph & $\mathrm{H}$ & $\begin{array}{l}290 \\
\text { dec. }\end{array}$ & 64 & 1713 & 1224 & 1669 & 1092 & $\begin{array}{l}3464 \\
3280\end{array}$ & $\begin{array}{l}312 \\
288\end{array}$ \\
\hline 54 & $3-\mathrm{NO}_{2} \mathrm{Ph}$ & $\mathrm{H}$ & $\begin{array}{l}240 \\
\text { dec. }\end{array}$ & 56 & 1685 & 1271 & 1647 & 1020 & 3475 & $\begin{array}{l}303 \\
286\end{array}$ \\
\hline 55 & 3-pyridyl & $\mathrm{H}$ & $\begin{array}{l}165- \\
167\end{array}$ & 72 & 1681 & 1278 & 1575 & 1085 & 3422 & $\begin{array}{l}312 \\
261\end{array}$ \\
\hline 56 & $\begin{array}{c}\text { Benzimidazol- } \\
2-y l\end{array}$ & $\mathrm{CH}_{3}$ & $\begin{array}{l}190 \\
\text { dec. }\end{array}$ & 52 & 1703 & 1282 & 1620 & 1012 & 3417 & $\begin{array}{l}336 \\
262\end{array}$ \\
\hline
\end{tabular}

The chalcones was treated with guanidine nitrate in the presence of sodium ethoxide to give the compounds[57-65]. The IR spectra of compounds [57-65] show absorption at (1172-1295)v $\mathrm{cm}^{-1}(\mathrm{C}-\mathrm{N})$ and $(1590-1655) \vee \mathrm{cm}^{-1}(\mathrm{C}=\mathrm{N})^{[32]}, \quad \lambda \neg \max (371-304 \mathrm{~nm})$ and $(311-214 \mathrm{~nm})$ 
(Table7). ${ }^{1} \mathrm{HNMR}$ [400MHz, $\delta$, ppm, DMSO-d ${ }^{6}$ ] of compounds[64] $3.391 \delta(2 \mathrm{H}, \mathrm{S},-\mathrm{CH} 2), 6.946 \delta(1 \mathrm{H}, \mathrm{b},-\mathrm{CH}), 7.245-7.330 \delta(2 \mathrm{H}, \mathrm{m}, \mathrm{Ar}-$ $\mathrm{H}), 7.567-7.630 \delta(2 \mathrm{H}, \mathrm{m}, \mathrm{Ar}-\mathrm{H}), \quad 7.753-7.772 \delta(1 \mathrm{H}, \mathrm{t}$, pyridine $) 8.016$ $\delta(1 \mathrm{H}, \mathrm{s}$, pyridine $), 8.521-8.540 \delta(1 \mathrm{H}, \mathrm{d}$, pyridine $), 8.733-8.744 \delta(1 \mathrm{H}, \mathrm{d}$, pyridine $), \quad 9.349 \delta(1 \mathrm{H}, \mathrm{S}, \mathrm{NH} 2), \quad 13.072 \delta(1 \mathrm{H}, \mathrm{S}, \mathrm{N}-\mathrm{H})$, the physical properties and spectral data are listed in Table (7).

$$
\begin{aligned}
& \stackrel{\stackrel{N}{\|}}{\mathrm{NH}_{2} \mathrm{CNH}_{2}} \cdot \mathrm{HNO}_{3}+\mathrm{KOH} \longrightarrow \stackrel{\stackrel{N}{\|}}{\mathrm{NH}_{2} \mathrm{CNH}_{2}}+\mathrm{KNO}_{3}+\mathrm{H}_{2} \mathrm{O} \\
& \text { (c) } \\
& \text { (n) } \\
& \text { (57-65] } \\
& \mathrm{Ar}=\mathrm{Ph} \quad, \quad 4-\left(\mathrm{N}, \mathrm{N}\left(\mathrm{CH}_{3}\right)_{2}\right) \mathrm{Ph}, \quad 4-\mathrm{OCH}_{3} \mathrm{Ph} \quad, \quad 3,4-\left(\mathrm{OCH}_{3}\right)_{2} \mathrm{Ph} \\
& \text { 2,6- }(\mathrm{Cl})_{2} \mathrm{Ph} \quad, \quad 4-\mathrm{Cl} \mathrm{Ph} \quad, \quad 3-\mathrm{NO}_{3} \mathrm{Ph} \quad, \quad 3 \text {-pyridyl , benzimidazol-2-yl } \\
& \mathrm{X}=\mathrm{H} \quad, \quad \mathrm{CH}_{3} \quad \text { (in compound [74]) }
\end{aligned}
$$

Scheme (6)

The suggested mechanism for the reaction of chalcones with guanidine nitrate 
Table(7): Physical properties and spectral data [IR (KBr) $v \mathrm{~cm}^{-1}$ and U.Vnm] of compounds [57-65]<smiles></smiles>

\begin{tabular}{|c|c|c|c|c|c|c|c|c|c|}
\hline \multirow{2}{*}{$\begin{array}{l}\text { Comp. } \\
\text { No }\end{array}$} & \multirow{2}{*}{$\mathrm{Ar}$} & \multirow{2}{*}{ X } & \multirow{2}{*}{$\begin{array}{l}\text { M.P } \\
\left({ }^{\circ} \mathrm{C}\right)\end{array}$} & \multirow{2}{*}{$\begin{array}{c}\text { Yiel } \\
d \\
(\%) \\
\end{array}$} & \multicolumn{4}{|c|}{ IR v $\mathrm{cm}^{-1}(\mathrm{KBr})$} & \multirow{2}{*}{$\begin{array}{c}\text { U.V } \\
\text { inDMF } \\
\lambda_{\max },(\mathrm{nm}) \\
\end{array}$} \\
\hline & & & & & $\mathrm{C}=\mathrm{N}$ & $\mathrm{C}=\mathrm{C}$ & $\mathrm{C}-\mathrm{N}$ & $\mathrm{NH}_{2}$ & \\
\hline 57 & $\mathrm{Ph}$ & $\mathrm{H}$ & $\begin{array}{l}257 \\
\text { dec. }\end{array}$ & 73 & 1655 & 1591 & 1172 & 3448 & $\begin{array}{l}r \leqslant v \\
r 11\end{array}$ \\
\hline 58 & $\varepsilon-\mathrm{N}, \mathrm{N}\left(\mathrm{CH}_{3}\right)_{2} \mathrm{Ph}$ & $\mathrm{H}$ & $\begin{array}{c}145- \\
148\end{array}$ & 69 & 1580 & 1540 & 1277 & $\begin{array}{l}3394 \\
3180\end{array}$ & $\begin{array}{l}\text { צII } \\
\text { TM }\end{array}$ \\
\hline 59 & $4-\mathrm{OCH}_{3} \mathrm{Ph}$ & $\mathrm{H}$ & $\begin{array}{l}110- \\
112\end{array}$ & 54 & 1591 & 1548 & 1295 & $\begin{array}{l}3471 \\
3392\end{array}$ & $\begin{array}{l}\text { TrY } \\
\text { Tru }\end{array}$ \\
\hline 60 & $3,4-\left(\mathrm{OCH}_{3}\right)_{2} \mathrm{Ph}$ & $\mathrm{H}$ & $\begin{array}{r}130- \\
133\end{array}$ & 70 & 1616 & 1580 & 1275 & $\begin{array}{l}3464 \\
3375\end{array}$ & $\begin{array}{l}r 09 \\
r \leqslant 1\end{array}$ \\
\hline 61 & $2,6-(\mathrm{Cl})_{2} \mathrm{Ph}$ & $\mathrm{H}$ & $\begin{array}{l}161- \\
163\end{array}$ & 57 & 1618 & 1589 & 1277 & $\begin{array}{l}3475 \\
3381\end{array}$ & $\begin{array}{l}r \cdot \varepsilon \\
r \mid \varepsilon\end{array}$ \\
\hline 62 & 4-Cl Ph & $\mathrm{H}$ & $\begin{array}{c}154- \\
157\end{array}$ & 67 & 1635 & 1595 & 1261 & 3437 & $\begin{array}{l}\text { TrI } \\
\text { rVI }\end{array}$ \\
\hline 63 & $3-\mathrm{NO}_{2} \mathrm{Ph}$ & $\mathrm{H}$ & $\begin{array}{l}294 \\
\text { dec. }\end{array}$ & 61 & 1590 & 1552 & 1237 & 3385 & $\begin{array}{l}\text { TOV } \\
\text { TVY }\end{array}$ \\
\hline 64 & 3-pyridyl & $\mathrm{H}$ & $\begin{array}{c}141- \\
143\end{array}$ & 58 & 1626 & 1590 & 1278 & $\begin{array}{l}3346 \\
3211\end{array}$ & $\begin{array}{l}r .1 \\
r . \varepsilon\end{array}$ \\
\hline 65 & Benzimidazol-2-yl & $\mathrm{CH}_{3}$ & $\begin{array}{l}167- \\
170\end{array}$ & 66 & 1676 & 1581 & 1281 & $\begin{array}{l}3410 \\
3292\end{array}$ & $\begin{array}{l}r . V \\
\text { roT }\end{array}$ \\
\hline
\end{tabular}

The chalcones was treated with ortho phenylenediamine in the presence of glacial acetic acid to give the benzdiazepines compound[6674]. The IR spectra ${ }^{[23]}$ of compounds [30-38] show absorption at(1620$1686 v \mathrm{~m}^{-1}(\mathrm{C}=\mathrm{N})$ and $\left(1253-1290 v \mathrm{~cm}^{-1}(\mathrm{C}-\mathrm{N}), \quad \lambda \neg \max (372-311 \mathrm{~nm})\right.$ and $(296-57 \mathrm{~nm})$ The physical properties and spectral data are listed in Table (8). 


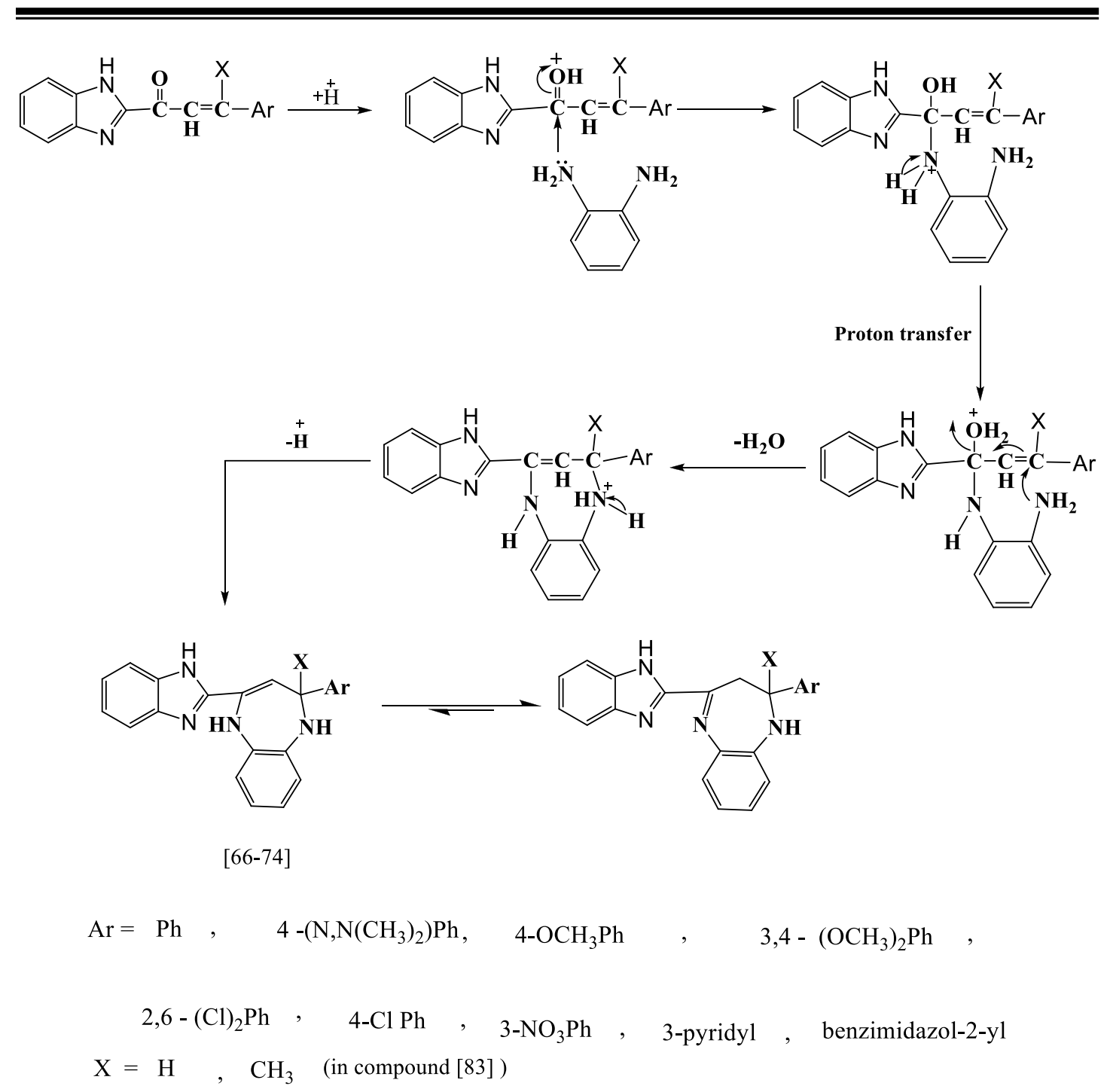

Scheme (7)

The suggested mechanism for the reaction of chalcones with ortho phenylenediamine 
Table(8):Physical properties and spectral data $\left[\mathrm{IR}(\mathrm{KBr}) v \mathrm{~cm}^{-1}\right.$ and U.Vnm] of compounds [66-74]

\begin{tabular}{|c|c|c|c|c|c|c|c|c|c|}
\hline \multirow{2}{*}{$\begin{array}{c}\text { Comp. } \\
\text { No }\end{array}$} & \multirow{2}{*}{$\mathrm{Ar}$} & \multirow{2}{*}{$\mathrm{X}$} & \multirow{2}{*}{$\begin{array}{l}\text { M.P } \\
\left({ }^{\circ} \mathrm{C}\right)\end{array}$} & \multirow{2}{*}{$\begin{array}{c}\text { Yiel } \\
\mathrm{d} \\
(\%)\end{array}$} & \multicolumn{4}{|c|}{ IR $v \mathrm{~cm}^{-1}(\mathrm{KBr})$} & \multirow{2}{*}{$\begin{array}{c}\text { U.V } \\
\text { inDMF } \\
\lambda_{\max },(\mathrm{nm}) \\
\end{array}$} \\
\hline & & & & & $\mathrm{C}=\mathrm{N}$ & $\mathrm{C}=\mathrm{C}$ & $\mathrm{N}-\mathrm{H}$ & C-N & \\
\hline 66 & $\mathrm{Ph}$ & $\mathrm{H}$ & $\begin{array}{c}116- \\
119\end{array}$ & 68 & 1686 & 1654 & 3358 & 1280 & $\begin{array}{l}372 \\
\text { rAr }\end{array}$ \\
\hline 67 & $\varepsilon-\mathrm{N}, \mathrm{N}\left(\mathrm{CH}_{3}\right)_{2} \mathrm{Ph}$ & $\mathrm{H}$ & $\begin{array}{l}150 \\
\text { dec. }\end{array}$ & 80 & 1638 & 1593 & 3427 & 1275 & $\begin{array}{l}\text { ror } \\
\text { rq1 }\end{array}$ \\
\hline 68 & $4-\mathrm{OCH}_{3} \mathrm{Ph}$ & $\mathrm{H}$ & $\begin{array}{c}135- \\
137\end{array}$ & 76 & 1682 & 1650 & 3380 & 1253 & $\begin{array}{l}\text { TYY } \\
\text { rVY }\end{array}$ \\
\hline 69 & $3,4-\left(\mathrm{OCH}_{3}\right)_{2} \mathrm{Ph}$ & $\mathrm{H}$ & $\begin{array}{c}196- \\
198\end{array}$ & 68 & 1664 & 1579 & 3255 & 1267 & $\begin{array}{l}\text { Tצד } \\
\text { T97 }\end{array}$ \\
\hline 70 & $2,6-(\mathrm{Cl})_{2} \mathrm{Ph}$ & $\mathrm{H}$ & $\begin{array}{c}136- \\
138\end{array}$ & 60 & 1661 & 1607 & rr85 & 1290 & $\begin{array}{l}\text { rov } \\
\text { rov }\end{array}$ \\
\hline 71 & 4-Cl Ph & $\mathrm{H}$ & $\begin{array}{c}180- \\
182\end{array}$ & 65 & 1647 & 1616 & 3242 & 1279 & $\begin{array}{l}\text { ror } \\
\text { rAt }\end{array}$ \\
\hline 72 & $3-\mathrm{NO}_{2} \mathrm{Ph}$ & $\mathrm{H}$ & $\begin{array}{r}134- \\
136\end{array}$ & 63 & 1664 & 1613 & 3384 & 1275 & $\begin{array}{l}\text { ril } \\
\text { rTo }\end{array}$ \\
\hline 73 & 3-pyridyl & $\mathrm{H}$ & $\begin{array}{l}240 \\
\text { dec. }\end{array}$ & 59 & 1624 & 1560 & 3215 & ITVV & $\begin{array}{l}\text { rov } \\
\text { rq4 }\end{array}$ \\
\hline 74 & Benzimidazol-2-yl & $\mathrm{CH}_{3}$ & $\begin{array}{c}153- \\
155\end{array}$ & 51 & 1620 & 1587 & 3113 & 1273 & $\begin{array}{l}\text { TYE } \\
\text { YAI }\end{array}$ \\
\hline
\end{tabular}

The chalcones was treated with 2-aminophenol in the presence of glacial acetic acid to give the benzoxazepines compound[75-83]. The IR spectra $^{[23]}$ of compounds [30-38] show absorption at (1086-7911) $v \mathrm{~cm}-1$ symmetrical and (1147-1255 ) v cm${ }^{-1}$ asymmetrical $(\mathrm{C}-\mathrm{O}-\mathrm{C})^{[27]},(1620$ - 
1686) $v \mathrm{~cm}^{-1}(\mathrm{C}=\mathrm{N}), \lambda \neg \max (368-268 \mathrm{~nm})$ and $(301-222 \mathrm{~nm})$, the physical properties and spectral data are listed in Table (9).

Table(9):Physical properties and spectral data [IR (KBr) $v \mathrm{~cm}^{-1}$ and U.Vnm] of compouns [75-83]<smiles>CC1(C)CC(c2nc3ccccc3[nH]2)=Nc2ccccc2O1</smiles>

\begin{tabular}{|c|c|c|c|c|c|c|c|c|c|}
\hline \multirow{2}{*}{$\begin{array}{c}\text { Comp. } \\
\text { No }\end{array}$} & \multirow{2}{*}{$\mathrm{Ar}$} & \multirow{2}{*}{$\mathrm{X}$} & \multirow{2}{*}{$\begin{array}{l}\text { M.P } \\
\left({ }^{\circ} \mathrm{C}\right)\end{array}$} & \multirow{2}{*}{$\begin{array}{l}\text { Yiel } \\
\mathrm{d} \\
(\%)\end{array}$} & \multicolumn{4}{|c|}{ IR $v \mathrm{~cm}^{-1}(\mathrm{KBr})$} & \multirow{2}{*}{$\begin{array}{c}\text { U.V } \\
\text { inDMF } \\
\lambda_{\max }, \\
(\mathrm{nm}) \\
\end{array}$} \\
\hline & & & & & $\mathrm{C}=\mathrm{N}$ & $\mathrm{C}-\mathrm{O}-\mathrm{C}$ & $\mathrm{N}-\mathrm{H}$ & $\mathrm{C}-\mathrm{N}$ & \\
\hline 75 & $\mathrm{Ph}$ & $\mathrm{H}$ & $\begin{array}{c}145- \\
147\end{array}$ & 74 & 1599 & $\begin{array}{l}\text { as1194 } \\
\text { sy1086 }\end{array}$ & 3284 & 1254 & $\begin{array}{l}344 \\
260\end{array}$ \\
\hline 76 & $\left\{-\mathrm{N}, \mathrm{N}\left(\mathrm{CH}_{3}\right)_{2} \mathrm{Ph}\right.$ & $\mathrm{H}$ & \begin{tabular}{|l|}
$283-$ \\
285 \\
\end{tabular} & 86 & 1634 & $\begin{array}{l}\text { as1231 } \\
\text { sy1179 }\end{array}$ & 3445 & 1281 & $\begin{array}{l}322 \\
278\end{array}$ \\
\hline 77 & $4-\mathrm{OCH}_{3} \mathrm{Ph}$ & $\mathrm{H}$ & \begin{tabular}{|c|}
$196-$ \\
198
\end{tabular} & 69 & 1685 & $\begin{array}{l}\text { as } 1255 \\
\text { sy1092 }\end{array}$ & 3255 & 1285 & $\begin{array}{l}362 \\
301\end{array}$ \\
\hline 78 & $3,4-\left(\mathrm{OCH}_{3}\right)_{2} \mathrm{Ph}$ & $\mathrm{H}$ & \begin{tabular}{|c|}
$198-$ \\
199 \\
\end{tabular} & 62 & 1655 & $\begin{array}{l}\text { as1215 } \\
\text { sy1101 }\end{array}$ & 3255 & 1273 & $\begin{array}{l}268 \\
222\end{array}$ \\
\hline 79 & $2,6-(\mathrm{Cl})_{2} \mathrm{Ph}$ & $\mathrm{H}$ & $\begin{array}{l}218- \\
220\end{array}$ & 65 & 1662 & $\begin{array}{l}\text { as } 1213 \\
\text { sy1140 }\end{array}$ & r 85 & 1327 & $\begin{array}{l}309 \\
240\end{array}$ \\
\hline 80 & 4-Cl Ph & $\mathrm{H}$ & \begin{tabular}{|c|}
$241-$ \\
243
\end{tabular} & 58 & 1657 & $\begin{array}{l}\text { as1215 } \\
\text { sy1138 }\end{array}$ & 3261 & 1290 & $\begin{array}{l}346 \\
269\end{array}$ \\
\hline 81 & $3-\mathrm{NO}_{2} \mathrm{Ph}$ & $\mathrm{H}$ & \begin{tabular}{|l|}
$216-$ \\
218
\end{tabular} & 71 & 1662 & $\begin{array}{l}\text { as1213 } \\
\text { sy1149 }\end{array}$ & 3290 & 1213 & $\begin{array}{l}359 \\
294\end{array}$ \\
\hline 82 & 3-pyridyl & $\mathrm{H}$ & \begin{tabular}{|c|}
$169-$ \\
171 \\
\end{tabular} & 55 & 1678 & $\begin{array}{l}\text { as1228 } \\
\text { sy1120 }\end{array}$ & 3180 & ITVV & $\begin{array}{l}317 \\
240\end{array}$ \\
\hline 83 & Benzimidazol-2-yl & $\mathrm{CH}_{3}$ & \begin{tabular}{|l|}
190 \\
dec. \\
\end{tabular} & 61 & 1676 & $\begin{array}{l}\text { as1147 } \\
\text { sy1086 }\end{array}$ & 3290 & 1236 & $\begin{array}{l}308 \\
248 \\
\end{array}$ \\
\hline
\end{tabular}









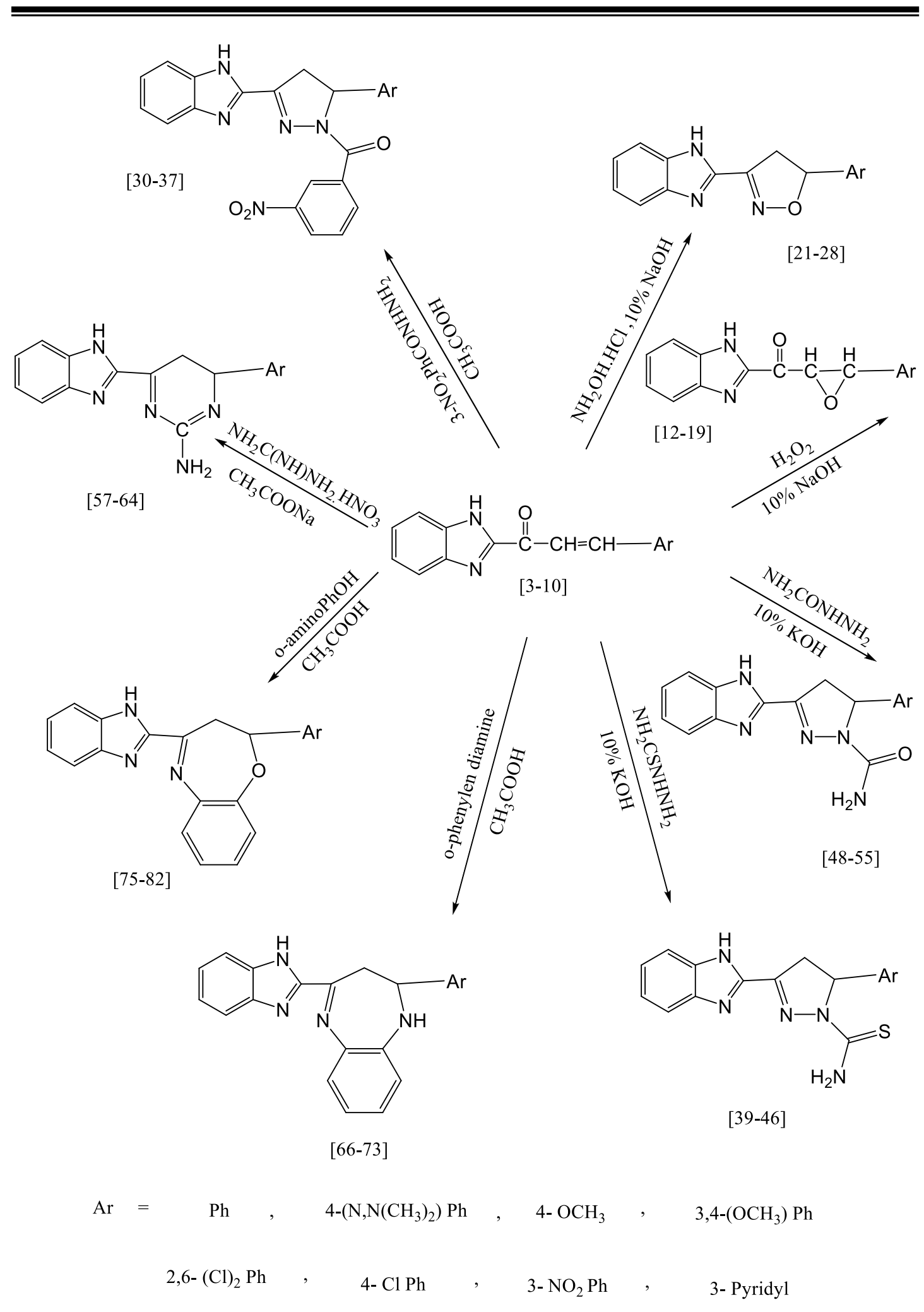

Scheme(9): The reaction route of chalcones [1-8] 


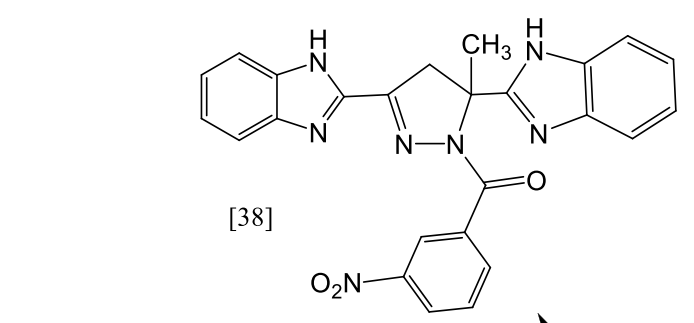

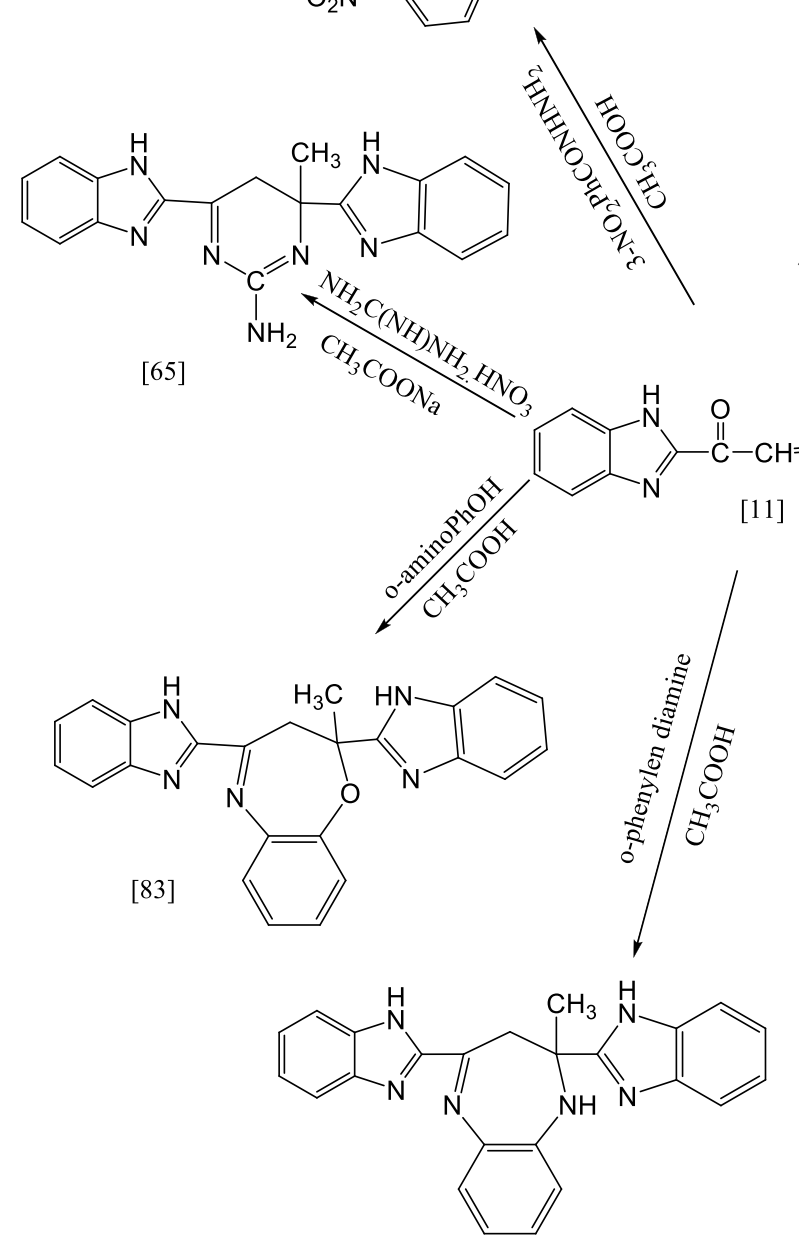

[74]

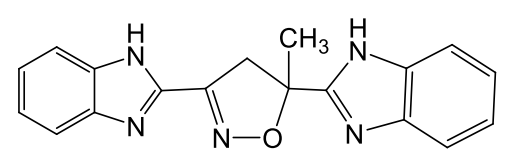

[29]
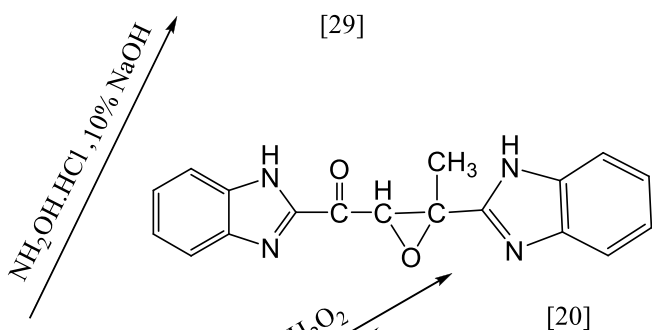

[20]<smiles>CCc1nc2ccccc2[nH]1</smiles>

$\mathrm{H}_{2} / \mathrm{OH}$

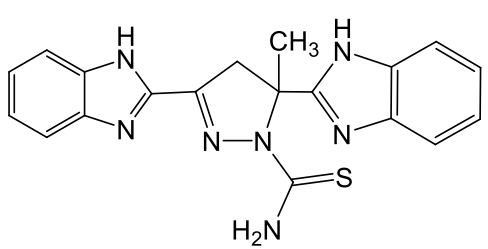

[47]

Scheme(10): The reaction route of chalcone [9] 


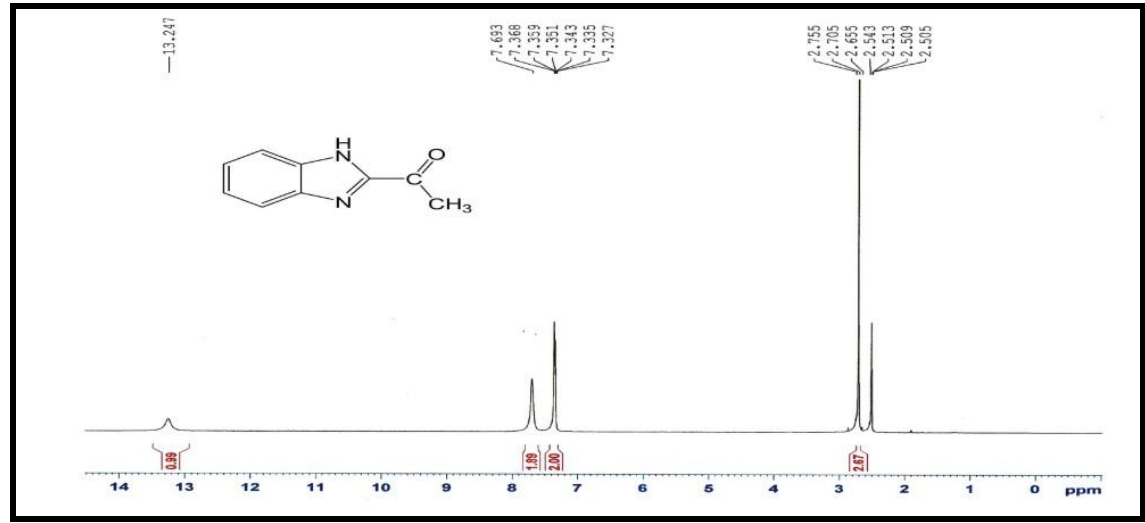

${ }^{1}$ HNMR of compound[2]


\section{REFERNCES}

1. HP Avila; EF Smania; FD Monache; AJr Smania., Bioorg. Med. Chem16, 9790. (2008).

2. J H Cheng; C F Hung; S C Yang; J P Wang; S J Won; C N Lin., Bioorg. Med.Chem., 16,7270,(2008).

3. S Murakami; M Muramatsu; H Aihara; S Otomo. Biochem. Pharmacol, 42, 1447-1451. (1991). 
4. GS Viana; MA Bandeira; FJ Matos. Phytomedicine., 10, 189-195, (2003).

5. JH Wu; XH Wang; YH Yi; K H Lee. Bioorg. Med. Chem. Lett., 13,1813-1815,(2003).

6. M Liu; P Go; MLWilairat. J. Med. Chem, 44, 4443-4452.(2001).

7. Lopez, S. N.; Castelli, M. V.; Zacchino, S. A.; Dominguez, J. N. Lobo, G.; Jaime, C. C, Cortes, J. C. G.; Ribas, J. C.; Devia, C. Ana, M. R.; Ricardo, D. E. Bioorg. Med. Chem. 2001, 9, (1999).

8. HJ Zhang; Y Qian; DD Zhu; X G Yang; HL Zhu. European Journal of Medicinal Chemistry, 46 4702-4708, (2011).

9. Balkrishna Tiwari, A S Pratapwar, A R Tapas, S R Butle, and BS Vatkar, Int.J. ChemTech Res.2010, 2(1).

10.Thanh-Dao Tran, Th Nhu Nguyen, Tuong-Ha Do, Khac-Minh Thai, and Cat-Dong Tran, International Electronic Conference on Synthetic Organic Chemistry,1-30, (2011).

11.S Zangade, S Mokle, A Vibhute, Y Vibhute, Chemical Sciences Journal, Volume, CSJ-13, (r+1)).

12.Gayathri.B, S.M.Hipparagi, Ramjith.U.S, Cyril Mathews Jacob, Ramjith U.S. et al. IJRPS, 2(3), 146-158, (2012).

13.P.Kishore Kumar \& P. K. Dubey, Der Pharma Chemica, 4 (3):1292-1295, (2012).

14. I. Sudeer Babu and S. Selvakumar, International Journal of Biological \& Pharmaceutical Research, 3(2): 275-284,(2012).

15. Anjani. S, Kishor. K, Ana. C, Marina.S, Irini.D and Athina Geronikaki, European Journal of Medicinal Chemistry, 45, 510-518, (2010)

16.Seema I. Habib and Praffullkumar A. Kulkarni, Der Pharmacia Lettre, 5 (2):101-104,(2013).

17.Cromwell N. H., Bamlurg R. E. and Bakly R. P., J. Am. Chem. Soc., 81, 4294,(1959).

18.Vijay kumar Tirlapur, Narasimha Gandhi, Raga Basawaraj and Rajendra Prasad Y. International Journal of ChemTech Research, Vol.2, No.3, pp 1434-1440,(2010).

19.Ch.Sridevi, K.Balaji, A.Naidu, S.Kavimani, D.Venkappayya, R.Suthakaran and Sudha parimala, International journal of pharm Tech Research,Vol.1,No.3,pp816-821,(2009).

20.Singh Vinayaditya, Argal Ameeta, Mishra Vikash, Raghuvanshi Ramsneh, Agnihotri Savita, Singh Vinayaditya et al. IJRPS,1(3), 125$146,(r \cdot 11)$.

21.J Venkatesan, Pandeya, D Selvakumar, Indian J Pharm Sci, 69: p586-589. (2007). 
22.Janardan Singh Yadav and Y.K. Srivastava, Rasayan J. Chem. Vol. 3,No.4,p 726-730 (2010).

23.N. Garg, T. Chandra, Archana, A. Jain, A. Kumar, European Journal of Medicinal Chemistry, 45, 1529-1535, (2010).

24.Ashish Kumar Tewari and Anil Mishra,Indian Journal of chemistry,Vol, 45B, pp.489-493, (2006).

25.Adel Kamal Khider, British Journal of Pharmacology and Toxicology 2(2): 92-96, (2011).

26.Farouq E. Hawaiz, Lana H. Chawishli, Diler D. Ghafur, MSc. Thesis Chemistry Department, College of Science Education, University of Salahaddin, (2009).

27. Parikh.V.M, "Absorption Spectroscopy of Organic Molecules", P: 535540,(1985).

28.Rajeev Bhimwal, Anil K Sharma, Ankit Jain, Journal of Advanced Pharmacy Education \& Research1(5) 251-258, (2011).

29. Ameen Ali Abu-Hashem and Ahmed S. Aly, Arch Pharm Res Vol 35, No 3, 437-445, (2012).

30.Ameen Ali Abu-Hashem and Ahmed S. Aly, Arch Pharm Res Vol 35, No 3, 437-445, (2012).

31.Anees A Siddiqui, Md. Azizur Rahman, Md. Shaharyar, Ravinesh Mishra. Chemical Sciences Journal, Volume: CSJ-8, (2010).

32. V. M. Barot \& B.G. Rathod, Asian Journal of Biochemical and Pharmaceutical Research Issue: 4, Vol. 2, (2012). 